H. Morikawa

Nagoya Math. J.

Vol. 80 (1980), 1-47

\title{
SOME ANALYTIC AND GEOMETRIC APPLICATIONS OF THE INVARIANT THEORETIC METHOD
}

\author{
HISASI MORIKAWA
}

Determinant is a most useful tool in every branch of mathematics, especially in linear mathematics. What kind of quantities do take a similar universal important role as determinant, in advanced branches of mathematics? In the present articles, showing the usefullness of semiinvariants in the classical invariant theory, we shall give a partial answer of the above question.

\section{Chapter 1. Binary semi-invariants and automorphic forms}

Let $A$ be a commutative algebra over a field $K$ of characteristic zero. $A$ is called an $s l(2, K)$-algebra, if there exists a Lie algebra homomorphism of $\operatorname{sl}(2, K)$ into the Lie algebra of derivations of $A$ over $K$. The most familier example of $s l(2, K)$-algebra is a polynomial algebra $K\left[\xi^{(0)}, \cdots, \xi^{(n)}\right]$ with Cayley-Aronhold's operators;

$$
\begin{aligned}
\mathscr{H} & =\sum_{\ell=0}^{n}(n-2 \ell) \xi^{(\ell)} \frac{\partial}{\partial \xi^{(\ell)}}, \\
\mathscr{D} & =\sum_{\ell=0}^{n} \ell \xi^{(\ell-1)} \frac{\partial}{\partial \xi^{(\ell)}}, \\
\Delta & =\sum_{\ell=0}^{n}(n-\ell) \xi^{(\ell+1)} \frac{\partial}{\partial \xi^{(\ell)}}
\end{aligned}
$$

on which classical theory on $n$-forms works.

In the present chapter we shall show another important example of $s l(2, K)$-algebra associating with formal power series with variable coefficients, which is applied to automorphic forms.

Received September 2, 1977. 


\section{§1. Binary semi-invariants}

1.1. Let $K$ be a field of characteristic zero, and let $w=\left(w_{1}, \cdots, w_{N}\right)$ be a vector with components in $K$ such that $w_{1}, \cdots, w_{N}$ are neither zero nor positive integers, so that the generalized binary coefficients

$$
\left(\begin{array}{c}
w_{j} \\
\ell
\end{array}\right)=\frac{w_{j}\left(w_{j}-1\right) \cdots\left(w_{j}-\ell+1\right)}{\ell !} \quad(\ell=0,1, \cdots ; 1 \leq j \leq N)
$$

never vanish. Let $\xi_{1}=\left(\xi_{1}^{(0)}, \xi_{1}^{(1)}, \xi_{1}^{(2)}, \cdots\right), \cdots, \xi_{N}=\left(\xi_{N}^{(0)}, \xi_{N}^{(1)}, \xi_{N}^{(3)}, \cdots\right)$ be vectors of infinite length with independent variable coefficients $\xi_{j}^{(\ell)}(\ell=0,1,2, \cdots$; $1 \leq j \leq N)$. Degree, weight and index are defined on the polynomial algebra

$$
K\left[\xi_{1}, \cdots, \xi_{N}\right]=K\left[\left(\xi_{j}^{(\ell)}\right)_{\ell=0,2,3, \cdots ; 1 \leq j \leq N}\right]
$$

as follows;

$$
\begin{aligned}
& \operatorname{deg}\left(\xi_{j}^{(\ell)}\right)=1, \quad \text { weight }\left(\xi_{j}^{(\ell)}\right)=\ell, \quad(\ell=0,1,2, \cdots ; 1 \leq j \leq N) \\
& \operatorname{index}\left(\xi_{j}^{(\ell)}\right)=w_{j}-2 \ell .
\end{aligned}
$$

Degree and weight are independent on the choice of $w=\left(w_{1}, \cdots, w_{N}\right)$, but index depends on $w=\left(w_{1}, \cdots, w_{N}\right)$.

A polynomial is called to be isobaric, if it is a sum of monomials of same weight, and a polynomial is called to be index-homogeneous, if it is a sum of monomials of same index.

Denoting by

$$
K\left[\xi_{1}, \cdots, \xi_{N}\right]^{[u]}
$$

the vector space of index homogeneous polynomials of index $u$, we get a direct sum decomposition, called index-decomposition,

$$
K\left[\xi_{1}, \cdots, \xi_{N}\right]=\bigoplus_{u} K\left[\xi_{1}, \cdots, \xi_{N}\right]^{[u]} .
$$

To make $K\left[\xi_{1}, \cdots, \xi_{N}\right]$ an $s l(2, K)$-algebra, we introduce Cayley-Aronhold's differential operators;

$$
\begin{aligned}
\mathscr{H} & =\sum_{j=1}^{N} \sum_{\ell=1}^{\infty}\left(w_{j}-2 \ell\right) \xi_{j}^{(\ell)} \frac{\partial}{\partial \xi^{(\ell)}} \\
\mathscr{D} & =\sum_{j=1}^{N} \sum_{\ell=0}^{\infty} \ell \xi_{j}^{(\ell-)} \frac{\partial}{\partial \xi_{j}^{(\ell)}}, \\
\Delta & =\sum_{j=1}^{N} \sum_{\ell=0}^{\infty}\left(w_{j}-\ell\right)_{j}^{(\ell+1)} \frac{\partial}{\partial \xi_{i}^{(\ell)}} .
\end{aligned}
$$


The next lemma shows that the action;

$$
\left[\begin{array}{rr}
-1 & 0 \\
0 & 1
\end{array}\right] \varphi=\mathscr{H} \varphi, \quad\left[\begin{array}{ll}
0 & 0 \\
1 & 0
\end{array}\right] \varphi=\mathscr{D} \varphi, \quad\left[\begin{array}{ll}
0 & 1 \\
0 & 0
\end{array}\right] \varphi=\Delta \varphi
$$

gives an $\operatorname{sl}(2, K)$-algebra structure on $K\left[\xi_{1}, \cdots, \xi_{N}\right]$.

\section{LEMMA 1.1.}

$$
[\mathscr{D}, \Delta]=\mathscr{H}, \quad[\mathscr{H}, \mathscr{D}]=2 \mathscr{D}, \quad[\mathscr{H}, \Delta]=-2 \Delta .
$$

Proof. It is enough to prove the equalities for the action on the generators $\xi_{j}^{(\ell)}$;

$$
\begin{aligned}
{[\mathscr{D}, \Delta] \xi_{j}^{(\ell)} } & =\left\{\left(w_{j}-\ell\right)(\ell+1)-\ell\left(w_{j}-\ell+1\right)\right\} \xi_{j}^{(\ell)} \\
& =\left(w_{j}-2 \ell\right) \xi_{j}^{(\ell)}=\mathscr{H} \xi_{j}^{(\ell)} \\
{[\mathscr{H}, \mathscr{D}] \xi_{j}^{(\ell)} } & =\left\{\ell\left(w_{j}-2 \ell+2\right)-\left(w_{j}-2 \ell\right) \ell\right\} \xi_{j}^{(\ell)} \\
& =2 \ell \xi_{j}^{(\ell-1)}=2 \mathscr{D} \xi_{j}^{(\ell)} \\
{[\mathscr{H}, \Delta] \xi_{j}^{(\ell)} } & =\left\{\left(w_{j}-\ell\right)\left(w_{j}-2 \ell-2\right)-\left(w_{j}-2 \ell\right)\left(w_{j}-\ell\right)\right\} \xi_{j}^{(\ell+1)} \\
& =-2\left(w_{j}-\ell\right) \xi_{j}^{(\ell+1)}=-2 \Delta \xi_{j}^{(\ell)} .
\end{aligned}
$$

The next lemma states that the index-decomposition

$$
K\left[\xi_{1}, \cdots, \xi_{N}\right]=\bigoplus_{u} K\left[\xi_{1}, \cdots, \xi_{N}\right]^{[u]}
$$

is the eigen-space decomposition with respect to operator $\mathscr{H}$.

LemMa 1.2. Let $\varphi$ be an index-homogeneous polynomial of index $u$. Then

$$
\mathscr{H} \varphi=u \varphi \text {. }
$$

Proof. It is enough to prove Lemma for a monomial $\phi$. Since

$$
\begin{aligned}
& \operatorname{deg}_{\xi_{j}}(\varphi) \phi=\left[\sum_{\ell=0}^{\infty} \xi_{j}^{(\ell)}\left[\sum_{\ell=0}^{\infty} \frac{\partial}{\partial \xi_{j}^{(\ell)}}\right] \phi,\right. \\
& {\left[\sum_{j=1}^{N} \sum_{\ell=0}^{n} \ell \xi_{j}^{(\ell)} \frac{\partial}{\partial \xi_{j}^{(\ell)}}\right] \phi=\text { weight }(\phi) \phi,}
\end{aligned}
$$

we have

$$
\begin{aligned}
\mathscr{H} \phi & =\left[\sum_{j=1}^{N} \sum_{\ell=0}^{\infty}\left(w_{j}-2 \ell\right) \frac{\partial}{\partial \xi_{j}^{(\ell)}}\right] \phi \\
& =\left[\sum_{j=1}^{N} w_{j} \operatorname{deg}(\phi)-2 \text { weight }(\omega)\right] \phi=\operatorname{index}(\phi) \phi .
\end{aligned}
$$


LEMMA 1.3.

$$
\begin{aligned}
& {\left[\mathscr{D}, \Delta^{\ell}\right]=\sum_{u} \ell(u-\ell+1) \Delta^{\ell-1} \pi^{[u]},} \\
& {\left[\Delta, \mathscr{D}^{\ell}\right]=\sum_{u} \ell(-u-\ell+1) D^{\ell-1} \pi^{[u]},}
\end{aligned}
$$

where $\pi^{[u]}$ is the projection $K\left[\xi_{1}, \cdots, \xi_{N}\right] \rightarrow K\left[\xi_{1}, \cdots, \xi_{N}\right]^{[u]}$.

Proof. We shall prove (1.3) by induction on $\ell$. For $\ell=1$ (1.3) coincides with (1.2). Assume that

$$
\left[\mathscr{D}, \Delta^{\ell-1}\right]=\sum_{u}(\ell-1)(u-\ell+2) \pi^{[u]} .
$$

Then we have

$$
\begin{aligned}
{\left[\mathscr{D}, \Delta^{\ell}\right] } & =\mathscr{D} \Delta \Delta^{\ell-1}-\Delta \mathscr{D} \Delta^{\ell-1}+\Delta \mathscr{D} \Delta^{\ell-1}-\Delta \Delta^{\ell-1} \mathscr{D} \\
& =\mathscr{H} \Delta^{\ell-1}+\Delta\left[\mathscr{D}, \Delta^{\ell-1}\right] \theta \\
& =\mathscr{H} \Delta^{\ell-1} \sum_{u} \pi^{[u]}+\Delta \sum_{u}(\ell-1)(u-\ell+2) \Delta^{\ell-2} \pi^{[u]} \\
& =\sum_{u}(u-2 \ell+2) \Delta^{\ell-1} \pi^{[u]}+\sum_{u}(\ell-1)(u-\ell+2) \Delta^{\ell-1} \pi^{[u]} \\
& =\sum_{u} \ell(u-\ell+1) \Delta^{\ell-1} \pi^{[u]} .
\end{aligned}
$$

(1.4) is also proved by the similar way.

LEMMA 1.4.

$$
\begin{aligned}
& \mathscr{D}^{\ell} K\left[\xi_{1}, \cdots, \xi_{N}\right]^{[u]} \subset K\left[\xi_{1}, \cdots, \xi_{N}\right]^{[u+2 \ell]} \quad(\ell=0,1,2, \cdots) \\
& \Delta^{\ell} K\left[\xi_{1}, \cdots, \xi_{N}\right]^{[u]} \subset K\left[\xi_{1}, \cdots, \xi_{N}\right]^{[u-2 \ell]}
\end{aligned}
$$

Proof. It is sufficient to prove $\ell=1$. For a monomial $\phi$ we have

$$
\begin{aligned}
& \operatorname{deg}_{\xi_{j}}(\mathscr{D} \phi)=\operatorname{deg}_{\xi_{j}}(\Delta \phi)=\operatorname{deg}_{\xi_{j}}(\phi), \\
& \text { weight }(\mathscr{D} \phi)=\text { weight }(\phi)-1, \text { weight }(\Delta \phi)=\text { weight }(\phi)+1,
\end{aligned}
$$

and thus

$$
\begin{gathered}
\operatorname{index}(\mathscr{D} \phi)=\sum_{j=1}^{N} w_{j} \operatorname{deg}_{\xi_{j}}(\phi)-2 \text { weight }(\phi)+2=\operatorname{index}(\phi)+2, \\
\operatorname{index}(\Delta \phi)=\sum_{j=1}^{N} w_{j} \operatorname{deg}_{\xi_{j}}(\phi)-2 \text { weight }(\phi)-2=\operatorname{index}(\phi)-2 .
\end{gathered}
$$

Definition 1.1. A polynomial $\varphi$ is called a binary semi-invariant if $\mathscr{D} \varphi=0$. We denote by $\mathfrak{S}$ the $K$-algebra of binary semi-invariants in $K\left[\xi_{1}, \cdots, \xi_{N}\right]$. 
Since $\mathscr{D}$ does not depends on the choice of $w=\left(w_{1}, \cdots, w_{N}\right)$, the algebra of binary semi-invariants $\subseteq$ is independent on $w=\left(w_{1}, \cdots, w_{N}\right)$.

LEMMA 1.5 .

$$
\mathfrak{S}=\bigoplus_{u} \mathfrak{S}^{[u]},
$$

where

$$
\mathfrak{S}^{[u]}=\widetilde{S} \cap K\left[\xi_{1}, \cdots, \xi_{N}\right]^{[u]}
$$

Proof. Let $\varphi$ be an element of $\subseteq$ and let

$$
\varphi=\sum_{u} \varphi_{u}\left(\varphi_{u} \in K\left[\xi_{1}, \cdots, \xi_{N}\right]^{[u]}\right)
$$

be the index-decomposition of $\varphi$. Then by virtue of (1.2) we have

$$
\begin{aligned}
\sum_{u} u \mathscr{D} \varphi_{u}= & \mathscr{D} \mathscr{H} \sum_{u} \varphi_{u}=\mathscr{D} \mathscr{H} \varphi=(\mathscr{D} \mathscr{H}-\mathscr{H} \mathscr{D}) \varphi \\
& =[\mathscr{D}, \mathscr{H}] \varphi=2 \mathscr{D} \varphi=0 .
\end{aligned}
$$

Since $\mathscr{D} \varphi_{u}$ are linearly independent, we have $u \mathscr{D} \varphi_{u}=0$. Hence $\mathscr{D} \varphi_{u}=0$ for $u \neq 0$, and thus

$$
\mathscr{D} \varphi_{0}=\mathscr{D} \varphi-\sum_{u \neq 0} \mathscr{D} \varphi_{u}=0
$$

The index-decomposition

$$
\mathfrak{S}=\sum_{u} \mathfrak{S}^{[u]}
$$

depends on the choice of $w=\left(w_{1}, \cdots, w_{N}\right)$.

1.2. We shall next show that the $K$-algebra of binary semi-invariants $\widetilde{S}$ is not so complicated.

Lemma 1.6. Denoting

$$
\phi_{j \ell}(\xi)=\sum_{p=0}^{\ell}(-1)^{p}\left(\begin{array}{l}
\ell \\
p
\end{array}\right) \xi_{j}^{(\ell-p)} \xi_{j}^{(1) p} \xi_{j}^{(0) \ell-p-1} \quad(\ell=2,3, \cdots ; 1 \leq j \leq N)
$$

We have

$$
\mathscr{D} \phi_{j \ell}=0
$$

and

$$
\begin{aligned}
& K\left[\xi_{j}^{(0)}, \xi_{j}^{(0)-1} \xi_{j}^{(1)}, \xi_{j}^{(2)}, \xi_{j}^{(3)}, \cdots\right] \\
& \quad=K\left[\xi_{j}^{(0)}, \xi_{j}^{(0)-1} \xi_{j}^{(1)}, \xi_{j}^{(0)-1} \phi_{j 2}, \xi_{j}^{(0)-2} \phi_{j 3}, \cdots\right]
\end{aligned}
$$


for each $1 \leq j \leq N$.

Proof. From the definition of $\mathscr{D}$ it follows

$$
\begin{aligned}
\mathscr{D} \phi_{j \ell}= & \sum_{p=0}^{\ell}(-1)^{p}\left(\begin{array}{l}
\ell \\
p
\end{array}\right)\left\{(\ell-p) \xi_{j}^{(\ell-p-1)} \xi_{j}^{(1) p} \xi_{j}^{(0) \ell-p-1}+p \xi_{j}^{(\ell-p)} \xi_{j}^{(1) p-1} \xi_{j}^{(0) \ell-p}\right\} \\
= & \ell \xi_{j}^{(0) \ell-1} \sum_{p=0}^{\ell-1}(-1)^{p}\left(\begin{array}{c}
\ell-1 \\
p
\end{array}\right) \xi_{j}^{(\ell-p-1)} \xi^{(1) p} \xi_{j}^{(0) \ell-1-p-1} \\
& +\ell \xi_{j}^{(0) \ell-1} \sum_{p=1}^{\ell}(-1)^{p}\left(\begin{array}{c}
\ell-1 \\
p
\end{array}\right) \xi_{j}^{(\ell-p)} \xi_{j}^{(1) p-1} \xi_{j}^{(0) \ell-1-p} . \\
= & 0 .
\end{aligned}
$$

From the equations

$$
\xi_{j}^{(\ell)}=\xi^{(0)-(\ell-1)} \phi_{j \ell}-\sum_{p=1}^{\ell}(-1)^{p}\left(\begin{array}{l}
\ell \\
p
\end{array}\right) \xi_{j}^{(\ell-p)}\left(\xi_{j}^{(0)-1} \xi_{j}^{(1)}\right)^{p}
$$

it follows

$$
\begin{aligned}
& K\left[\xi_{j}^{(0)}, \xi_{j}^{(0)-1} \xi_{j}^{(1)}, \xi_{j}^{(2)}, \xi_{j}^{(3)}, \cdots\right] \\
& \quad=K\left[\xi_{j}^{(0)}, \xi_{j}^{(0)-1}, \xi_{j}^{(0)-1} \phi_{j 2}, \xi_{j}^{(0)-2} \phi_{j 3}, \cdots\right]
\end{aligned}
$$

Proposition 1.1.

$$
K\left[\xi_{1}, \cdots, \xi_{N}, \xi_{1}^{(0)-1}, \cdots, \xi_{n}^{(0)-1}\right]=\subseteq\left[\xi_{1}^{(0)-1}, \cdots, \xi_{N}^{(0)-1}, \xi_{1}^{(1)}\right] .
$$

Proof. From the definition of $\mathscr{D}$ it follows

$$
\mathscr{D}\left(\xi_{i}^{(1)} \xi_{j}^{(0)}-\xi_{i}^{(0)} \xi_{j}^{(1)}\right)=\xi_{i}^{(0)} \xi_{j}^{(0)}-\xi_{i}^{(0)} \xi_{j}^{(0)}=0 \quad(1 \leq i, j \leq N) .
$$

This means that

$$
\xi_{j}^{(1)} \in S_{[}\left[\xi_{1}^{(0)-1}, \cdots, \xi_{N}^{(0)-1}, \xi_{1}^{(1)}\right], \quad(1 \leq j \leq N) .
$$

By virtue Lemma 1.6

$$
\xi_{j}^{(\ell)}=\xi_{j}^{(0)-(\ell-1)} \phi_{j \ell}-\sum_{p=1}^{\ell}(-1)^{p}\left(\begin{array}{l}
\ell \\
p
\end{array}\right) \xi_{j}^{(\ell-p)}\left(\xi_{j}^{(0)-1} \xi_{j}^{(1)}\right)^{p}
$$

and $\mathscr{D} \phi_{j \ell}=0$, hence by induction on $\ell$ we have

$$
\xi_{j}^{(\ell)} \in \subseteq\left[\xi_{1}^{(0)-1}, \cdots, \xi_{N}^{(0)-1}, \xi_{1}^{(1)}\right] .
$$

1.3. The following formal power series with coefficients $\xi_{j}^{(\ell)}$

$$
f_{j}\left(\xi_{j} \mid z\right)=\sum_{\ell=0}^{\infty}\left(\begin{array}{c}
w_{j} \\
\ell
\end{array}\right) \xi_{j}^{(\ell)} z^{\ell} \quad(1 \leq j \leq N)
$$


are called the basic power series, and $K\left[\xi_{1}, \cdots, \xi_{N}\right]$ is called the $K$-algebra of coefficients of the basic formal power series. We denote briefly

$$
f_{j}^{(\ell)}\left(\xi_{j} \mid z\right)=\left(\frac{d}{d z}\right)^{\ell} f_{j}\left(\xi_{j} \mid z\right) \quad(\ell=0,1,2, \cdots ; 1 \leq j \leq N) .
$$

Let $y_{1}, \cdots, y_{N}$ be dependent variables with respect to the independent variable $z$. We denote briefly

$$
y_{j}^{(\ell)}=\left(\frac{d}{d z}\right)^{\ell} y_{j} \quad(\ell=0,1,2, \cdots ; 1 \leq j \leq N) .
$$

An element of $K\left[\left(y_{j}^{(\ell)}\right)_{\ell=0,1,2, \ldots ; 1 \leq j \leq N}\right]$ is called a differential polynomial, and an ideal $\mathfrak{a}$ is called a differential ideal if $(d / d z) \mathfrak{a} \subset \mathfrak{a}$. Since

$$
\left(\xi_{j}^{(\ell)}\right)_{\ell=0,1,2, \cdots ; 1 \leq j \leq N}, \quad\left(y_{j}^{(\ell)}\right)_{\ell=0,1,2, \cdots ; 1 \leq j \leq N}, \quad\left(f_{j}^{(\ell)}\left(\xi_{j} \mid z\right)\right)_{\ell=0,1,2, \cdots ; 1 \leq j \leq N}
$$

are three sets of algebraically independent quantities over $K$, there exist $K$-algebra isomorphisms $\Theta_{w}, \Phi_{w}$ and $\Psi$ :

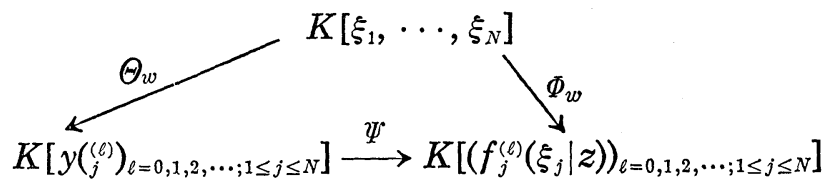

such that

$$
\begin{aligned}
\Theta_{w}\left(\xi_{j}^{(\ell)}\right) & =\frac{1}{w_{j}\left(w_{j}-1\right) \cdots\left(w_{j}-\ell+1\right)} y_{j}^{(\ell)}, \\
\Phi_{w}\left(\xi_{j}^{(\ell)}\right) & =\frac{1}{w_{j}\left(w_{j}-1\right) \cdots\left(w_{j}-\ell+1\right)} f_{j}^{(\ell)}\left(\xi_{j} \mid z\right), \\
\Psi\left(y_{j}^{(\ell)}\right) & =f_{j}^{(\ell)}\left(\xi_{j} \mid z\right) \quad(\ell=0,1,2, \cdots ; 1 \leq j \leq N) .
\end{aligned}
$$

LEMMA 1.7.

$$
\Phi_{w}(\varphi)=\exp (z \Delta) \varphi(\xi)=\sum_{\ell=0}^{\infty} \frac{1}{\ell !} \Delta^{\ell} \varphi(\xi) z^{\ell}
$$

Proof. From Leibniz's rule

$$
\Delta^{k}(\varphi \phi)=\sum_{\ell=0}^{k}\left(\begin{array}{l}
k \\
\ell
\end{array}\right) \Delta^{k-\ell} \varphi \Delta^{\ell} \phi
$$

it follows 


$$
\begin{aligned}
\exp (z \Delta)(\varphi \phi) & =\sum_{k=0}^{\infty} \frac{1}{k !} \Delta^{k}(\varphi \phi) z^{k}=\sum_{k=0}^{\infty} \frac{z^{k}}{k !} \sum_{\ell+h=k}\left(\begin{array}{l}
k \\
\ell
\end{array}\right) \Delta^{\ell} \varphi \Delta^{h} \phi \\
& =\left(\sum_{\ell=0}^{\infty} \frac{1}{\ell !} \Delta^{\ell} \varphi \cdot z^{\ell}\right)\left(\sum_{h=0}^{\infty} \frac{1}{h !} \Delta^{h} \phi z^{h}\right) \\
& =\exp (z \Delta) \varphi \exp (z \Delta) \phi .
\end{aligned}
$$

Obviously $\exp (z \Delta)$ is a linear operator, hence $\exp (z \Delta)$ is a $K$-algebra homomorphism of $K\left[\xi_{1}, \cdots, \xi_{N}\right]$ into $K\left[\left(f_{j}^{(\ell)}(\xi \mid z)\right)_{\ell=0,1,2, \cdots ; 1 \leq j \leq N}\right]$. Therefore it is enough to show

$$
\Phi_{w}\left(\xi_{j}^{(\ell)}\right)=\exp (z \Delta) \xi_{j}^{(\ell)} \quad(\ell=0,1,2, \cdots ; 1 \leq j \leq N) .
$$

From the definition of $\Phi_{w}$ it follows

$$
\begin{aligned}
\Phi_{w}\left(\xi_{j}^{(\ell)}\right) & =\frac{1}{w_{j}\left(w_{j}-1\right) \cdots\left(w_{j}-\ell+1\right)} f_{j}^{(\ell)}\left(\xi_{j} \mid z\right) \\
& =\frac{1}{w_{j}\left(w_{j}-1\right) \cdots\left(w_{j}-\ell+1\right)}\left(\frac{d}{d z}\right)^{\ell} \sum_{h=0}^{\infty}\left(\begin{array}{c}
w_{j} \\
h
\end{array}\right) \xi_{j}^{(h)} z^{h} \\
& =\sum_{h=0}^{\infty} \frac{1}{h !}\left(w_{j}-\ell\right)\left(w_{j}-\ell-1\right)\left(w_{j}-\ell-h+1\right) \xi_{j}^{(\ell+h)} z^{h} \\
& =\sum_{h=0}^{\infty} \frac{1}{h !} \Delta^{h} \xi_{j}^{(\ell)} z^{h} \\
& =\exp (z \Delta) \xi_{j}^{(\ell)} .
\end{aligned}
$$

LEMMA 1.8.

$$
\begin{aligned}
& \frac{d}{d z} \circ \Theta_{w}=\Theta_{w} \circ \Delta, \\
& \frac{d}{d z} \circ \Phi_{w}=\Phi_{w} \circ \Delta .
\end{aligned}
$$

Proof. From the definition of $\Theta_{w}$ and $\Delta$ it follows

$$
\begin{aligned}
\frac{d}{d z}\left(\Theta_{w} \xi_{j}^{(\ell)}\right) & =\frac{1}{w_{j}\left(w_{j}-1\right) \cdots\left(w_{j}-\ell+1\right)} \frac{d}{d z} y_{j}^{(\ell)} \\
& =\frac{1}{w_{j}\left(w_{j}-1\right) \cdots\left(w_{j}-\ell\right)} y_{j}^{(\ell+1)} \\
& =\left(w_{j}-\ell\right) \Theta_{w} \xi_{j}^{(\ell+1)}=\Theta_{w}\left(\Delta \xi_{j}^{(\ell)}\right) .
\end{aligned}
$$

Since $\xi_{j}^{(\ell)}(\ell=0,1,2, \cdots ; 1 \leq j \leq N)$ are generators of $K\left[\xi_{1}, \cdots, \xi_{N}\right]$, we have $d / d z \circ \Theta_{w}=\Theta_{w} \circ \Delta$. Similarly we can prove $d / d z \circ \Phi_{w}=\Phi_{w} \circ \Delta$.

By virtue of (1.14) an ideal $\mathfrak{a}$ of $K\left[\xi_{1}, \cdots, \xi_{N}\right]$ corresponds to a dif- 
ferential ideal by $\Theta_{w}$, if and only if $\mathfrak{a}$ is $\Delta$-admissible, i.e. $\Delta \mathfrak{a} \subset \mathfrak{a}$.

Definition 1.2. Let $A$ be a commutative $K$-algebra and let $a_{j}^{(\ell)}$ ( $\ell=$ $0,1,2, \cdots ; 1 \leq j \leq N$ ) be elements of $A$. For the system of formal power series

$$
f_{j}\left(a_{j} \mid z\right)=\sum_{\ell=0}^{\infty}\left(\begin{array}{c}
w_{j} \\
\ell
\end{array}\right) a_{j}^{(\ell)} z^{\ell} \quad(1 \leq j \leq N)
$$

with coefficients $a_{j}^{(\ell)}$ we associate two ideals; the kernel $\mathfrak{a}$ of the homomorphism of $K\left[\xi_{1}, \cdots, \xi_{N}\right]$ into $A$ such that

$$
\xi_{j}^{(\ell)} \longrightarrow a_{j}^{(\ell)} \quad(\ell=0,1,2, \cdots ; 1 \leq j \leq N)
$$

and the kernel $\mathfrak{A}$ of the homomorphism of $K\left[\left(y_{j}^{(\ell)}\right)_{\ell=0,1,2, \cdots ; 1 \leq j \leq N}\right]$ into $K\left[\left(f_{j}^{(\ell)}\left(a_{j} \mid z\right)\right)_{\ell=0,1,2, \ldots ; 1 \leq j \leq N}\right]$ such that

$$
y_{j}^{(\ell)} \longrightarrow f_{j}^{(\ell)}\left(a_{j} \mid z\right) \quad(\ell=0,1,2, \cdots ; 1 \leq j \leq N) .
$$

We call $\mathfrak{a}$ and $\mathfrak{A}$ respectively the coefficient ideal and the differential ideal of the system $\left(f_{1}\left(a_{1} \mid z\right), \cdots, f_{N}\left(a_{N} \mid z\right)\right)$ of formal power series.

Proposition 1.2. Let $\mathfrak{a}$ and $\mathfrak{U}$ be the coefficient ideal and the differential ideal of a system of formal power series

$$
f_{j}\left(a_{j} \mid z\right)=\sum_{\ell=0}^{\infty}\left(\begin{array}{c}
w_{j} \\
\ell
\end{array}\right) a_{j}^{(\ell)} z^{\ell} \quad(1 \leq j \leq N) .
$$

Let $\mathfrak{a}^{*}$ be the largest $\Delta$-admissible ideal contained in $\mathfrak{a}$. Then $\Theta_{w}\left(\mathfrak{a}^{*}\right)=\mathfrak{X}$.

Proof. By virtue of (1.13) it follows that $\Theta_{w}(\varphi)$ belongs to the differential ideal $\mathfrak{A}$, if and only if

$$
\begin{aligned}
0 & =\left.\Phi_{w}(\varphi)\right|_{\xi_{j}=a_{j}}=\left.\exp (z \Delta) \varphi(\xi)\right|_{\xi_{j}=a_{j}} \\
& =\sum_{\ell=0}^{\infty} \frac{1}{\ell !} \Delta^{\ell} \varphi\left(a_{j}\right) z^{\ell} .
\end{aligned}
$$

This means that $\Theta_{w}(\varphi) \in \mathfrak{a}$, if and only if $\Delta^{\ell} \varphi \in \mathfrak{a}(\ell=0,1,2, \cdots)$. On the other hand $\mathfrak{a}^{*}=\left\{\varphi \mid \Delta^{\ell} \varphi \in \mathfrak{a}, \ell=0,1,2, \cdots\right\}$ is the largest $\Delta$-admissible ideal contained in $a$.

Proposition 1.3. Let $\mathfrak{p}$ be a prime ideal of $K\left[\xi_{1}, \cdots, \xi_{n}\right]$, and let $\mathfrak{p}^{*}$ be the largest $\Delta$-admissible ideal contained in $\mathfrak{p}$. Then $\mathfrak{p}^{*}$ is also prime.

Proof. Denote $a_{j}^{(\ell)}$ the class of $\xi_{j}^{(\ell)}$ module $\mathfrak{p}$ and put 


$$
f_{j}^{(\ell)}\left(a_{j} \mid z\right)=\left(\frac{d}{d z}\right)^{\ell} \sum_{h=0}^{\infty}\left(\begin{array}{c}
w_{1} \\
h
\end{array}\right) a_{j}^{(h)} z^{h} \quad(\ell=0,1,2, \cdots ; 1 \leq j \leq N) .
$$

By virtue of Proposition 1.2 $\Phi_{w}$ induces an isomorphism

$$
K\left[\xi_{1}, \cdots, \xi_{N}\right] / p^{*} \simeq K\left[\left(f_{j}^{(\ell)}\left(a_{j} \mid z\right)\right)_{\ell=0,1,2, \cdots ; 1 \leq j \leq N}\right] .
$$

Since $\mathfrak{p}$ is prime, the residue algebra $K\left[\xi_{1}, \cdots, \xi_{N}\right] / \mathfrak{p}$ is an integral domain, hence $\left.K\left[f_{j}^{(\ell)}\left(a_{j} \mid z\right)\right)_{\ell=0,1,2, \ldots, 1 \leq j \leq N}\right]$ is also an integral domain. Then shows that $\mathfrak{p}^{*}$ is an prime ideal.

$\S 2$. Infinite dimensional representations of $\operatorname{sl}(2, K)$ and the decomposition of $K\left[\xi_{1}, \cdots, \xi_{N}\right]$,

2.1. For each element $u$ of $K$ we shall construct an infinite dimensional $\operatorname{sl}(2, K)$-module $W_{u}$, which is irreducible for $u \neq 0,1,2, \cdots$ and reducible for $u=0,1,2, \cdots$.

Proposition 1.4. Let $\left\{e_{\ell} \mid \ell=0,1,2, \cdots\right\}$ be a base of a vector space $W_{u}$ of infinite dimension over $K$, on which

$$
\left(\begin{array}{rr}
-1 & 0 \\
0 & 1
\end{array}\right), \quad\left(\begin{array}{ll}
0 & 0 \\
1 & 0
\end{array}\right), \quad\left(\begin{array}{ll}
0 & 1 \\
0 & 0
\end{array}\right)
$$

acts as follows

$$
\begin{aligned}
& \left(\begin{array}{rr}
-1 & 0 \\
0 & 1
\end{array}\right) e_{\ell}=(u-2 \ell) e_{\ell}, \\
& \left(\begin{array}{ll}
0 & 0 \\
1 & 0
\end{array}\right) e_{\ell}=(u-\ell+1) e_{\ell-1} \quad(\ell=0,1,2, \cdots), \\
& \left(\begin{array}{ll}
0 & 1 \\
0 & 0
\end{array}\right) e_{\ell}=(\ell+1) e_{\ell+1}
\end{aligned}
$$

where $e_{-1}=0$. Then the linear extension of these actions to $\operatorname{sl}(2, K)$ gives an $\operatorname{sl}(2, K)$-module structure on $W_{u}$. If $u \neq 0,1,2, \cdots$, then $W_{u}$ is irreducible, and if $u=0,1,2, \cdots$, the vector space $\tilde{W}_{u}$ generated by $\left\{e_{n+\ell+1} \mid \ell=\right.$ $0,1,2, \cdots\}$ is an irreducible $\operatorname{sl}(2, K)$-module isomorphic to $W_{-u-2}$ such that $W_{u} / \tilde{W}_{u}$ is an irreducible sl( $\left.2, K\right)$-module of dimension $u+1$.

Proof. From the definitions of the actions it follows

$$
\begin{aligned}
\left(\left(\begin{array}{ll}
0 & 0 \\
1 & 0
\end{array}\right),\left(\begin{array}{ll}
0 & 1 \\
0 & 0
\end{array}\right)\right) e_{\ell} & =\{(\ell+1)(u-\ell)-(u-\ell+1) e\} e_{\ell} \\
& =(u-2 \ell) e_{\ell}=\left(\begin{array}{rr}
-1 & 0 \\
0 & 1
\end{array}\right) e_{\ell},
\end{aligned}
$$




$$
\begin{aligned}
\left(\left(\begin{array}{rr}
-1 & 0 \\
0 & 1
\end{array}\right),\left(\begin{array}{ll}
0 & 0 \\
1 & 0
\end{array}\right)\right) e_{\ell} & =\{(u-\ell+1)(u-2 \ell-2)-(u-2 \ell)(u-\ell+1)\} e_{\ell} \\
& =2\left(\begin{array}{ll}
0 & 0 \\
1 & 0
\end{array}\right) e_{\ell} \\
\left(\left(\begin{array}{rr}
-1 & 0 \\
0 & 1
\end{array}\right),\left(\begin{array}{ll}
0 & 1 \\
0 & 0
\end{array}\right)\right) e_{\ell} & =\{(\ell+1)(u-2 \ell-2)-(u-2 \ell)(\ell+1)\} e_{\ell+1} \\
& =-2\left(\begin{array}{ll}
0 & 0 \\
0 & 1
\end{array}\right) e_{\ell} .
\end{aligned}
$$

This means that $W_{u}$ is an $s l(2, K)$-module. Denoting $P=\left\{a \in W_{u} \mid\left(\begin{array}{ll}0 & 0 \\ 1 & 0\end{array}\right) a=0\right\}$, we have

$$
P= \begin{cases}K e_{0} & \text { for } u \neq 0,1,2, \cdots \\ K e_{0}+K e_{u+1} & \text { for } u=0,1,2, \cdots,\end{cases}
$$

because $u-\ell+1=0$ if and only if $\ell=u+1$. Let $V$ be a non-zero $\operatorname{sl}(2, K)$-submodule of $W_{u}$. For each non-zero element a there exists a positive integer $n$ such that $\left(\begin{array}{ll}0 & 0 \\ 1 & 0\end{array}\right)^{n} a=0$. This means that $V \ddot{\cap} \dot{P}=\{0\}$. Hence, if $u \neq 0,1,2, \cdots$, then $V \ni e_{0}$ and $V=W_{u}$, i.e. $V$ is irreducible. If $u=0,1,2, \cdots$, then $\tilde{W}_{u}$ is an $s l(2, K)$-module isomorphic to $W_{-u-2}$, because

$$
\left(\begin{array}{rr}
-1 & 0 \\
0 & 1
\end{array}\right) e_{u+1}=(u-2 u-2) e_{u+1}=(-u-2) e_{u+1}
$$

The quotient $W_{u} / \tilde{W}_{u}$ is the known irreducible $s l(2, K)$-module of dimension $u+1$.

2.2. To proof semi-simplicity of $\operatorname{sl}(2, K)$-module $K\left[\xi_{1}, \cdots, \xi_{N}\right]$, it is necessary the next condition;

Condition (C): The set $\left\{\sum_{j=1}^{N} w_{j} d_{j} \mid d_{1}, \cdots, d_{N}\right.$ are not all zero non-negative integers

contains neither zero nor positive integer.

Theorem 1.1. If $w=\left(w_{1}, \cdots, w_{N}\right)$ satisfies Condition (C), then sl(2,K)module $K\left[\xi_{1}, \cdots, \xi_{N}\right]$ is semi-simple and $K\left[\xi_{1}, \cdots, \xi_{N}\right]$ is generated by binary semi-invariants as a sl(2,K)-module.

Proof. For each non-zero element $\varphi$ in $\mathfrak{S}^{[u]}$ putting

$$
\varphi^{(\ell)}=\frac{1}{\ell !} \Delta^{\ell} \varphi \quad(\varphi=0,1,2, \cdots),
$$


we have from (1.3)

$$
\begin{aligned}
\mathscr{H} \varphi^{(\ell)} & =(u-2 \ell) \varphi^{(\ell)}, \mathscr{D} \varphi^{(\ell)}=\frac{1}{\ell !} \mathscr{D} \Delta^{\ell} \varphi=\frac{\ell(u-\ell+1)}{\ell !} \Delta^{\ell-1} \varphi \\
& =(u-\ell+1) \varphi^{(\ell-1)} \\
\Delta \varphi^{(\ell)} & =\frac{1}{\ell !} \Delta^{\ell+1} \varphi=(\ell+1) \varphi^{(\ell+1)} .
\end{aligned}
$$

This shows that the vector subspace is an $\operatorname{sl}(2, K)$-module isomorphic to $W_{u}$; By virtue of Condition (C), the $s l(2, K)$-module

$$
W_{\varphi}=\bigoplus_{\ell=0}^{\infty} K \Delta^{\ell} \varphi
$$

is an irreducible $s l(2, K)$-module. Let $U$ be the $s l(2, K)$-submodule of $K\left[\xi_{1}, \cdots, \xi_{N}\right]$ generated by binary semi-invariants. Then $U$ is a semi-simple $\operatorname{sl}(2, K)$-module. Therefore it is enough to show $K\left[\xi_{1}, \cdots, \xi_{N}\right]=U$. Let $U^{[u]}$ be the vector subspace of $K\left[\xi_{1}, \cdots, \xi_{N}\right]$ generated by $\Delta^{e} \overleftarrow{S}^{[u+2 \ell]}(\ell=$ $0,1,2, \cdots)$, i.e.

$$
U^{[u]}=U \cap K\left[\xi_{1}, \cdots, \xi_{N}\right]^{[u]} .
$$

It is sufficient to show

$$
U^{[u]} \supset K\left[\xi_{1}, \cdots, \xi_{N}\right]^{[u]} \text {. }
$$

Since each element $\varphi$ in $K\left[\xi_{1}, \cdots, \xi_{N}\right]$ there exists a non-negative integer $n$ such that $\mathscr{D}^{n} \varphi \in \mathfrak{S}$, it is enough to prove the next assertion;

(*) If $\mathscr{D} \varphi \in U^{[u+2]}$, then $\varphi \in U^{[u]}$.

Case i) $u \neq-2,-3,-4, \cdots$ Let $\varphi$ be an element of $K\left[\xi_{1}, \cdots, \xi_{N}\right]^{[u]}$ such that $\mathscr{D} \varphi \in U^{[u+2]}$, and put

$$
\phi=\varphi+\sum_{\ell=1}^{\infty} \frac{(-1)^{\ell} \Delta^{\ell} \mathscr{D}^{\ell} \varphi}{\ell !(u+2)(u+3) \cdots(u+\ell+1)} .
$$

Then from (1.3) we have

$$
\begin{aligned}
\mathscr{D} \phi= & \mathscr{D} \varphi+\sum_{\ell=1}^{\infty} \frac{(-1)^{\ell} \mathscr{D} \Delta^{\ell} \mathscr{D}^{\ell} \varphi}{\ell !(u+2)(u+3) \cdots(u+\ell+1)} \\
= & \mathscr{D} \varphi+\sum_{\ell=1}^{\infty} \frac{(-1)^{\ell} \Delta^{\ell} \mathscr{D}^{\ell+1} \varphi}{\ell !(u+2)(u+3) \cdots(u+\ell+1)} \\
& +\sum_{\ell=1}^{\infty} \frac{(-1)^{\ell}\left[\mathscr{D}, \Delta^{\ell}\right] \mathscr{D}^{\ell} \varphi}{\ell !(u+2)(u+3) \cdots(u+\ell+1)}
\end{aligned}
$$




$$
\begin{aligned}
= & \mathscr{D} \varphi+\sum_{\ell=1}^{\infty} \frac{(-1)^{\ell} \Delta^{\ell} \mathscr{D}^{\ell+1} \varphi}{\ell !(u+2)(u+3) \cdots(u+\ell+1)} \\
& +\sum_{\ell=1}^{\infty} \frac{(-1)^{\ell} \ell(u+2 \ell-\ell+1) \Delta^{\ell-1} D^{\ell} \varphi}{\ell !(u+2)(u+3) \cdots(u+\ell+1)} \\
= & 0,
\end{aligned}
$$

namely $\phi \in \mathbb{S}^{[u]}$. Hence

$$
\varphi=\phi-\sum_{\ell=1}^{\infty} \frac{(-1)^{\ell} \Delta^{\ell} \mathscr{D}^{\ell} \varphi}{\ell !(u+2)(u+3) \cdots(u+\ell+1)} \in U^{[u]} .
$$

Case ii) $u=-2,-3,-4, \cdots$ Putting $n=-u-2$, with a nonnegative integer $n$. Let $\varphi$ be an element $\varphi$ of $K\left[\xi_{1}, \cdots, \xi_{N}\right]^{[u]}$ such that $\mathscr{D} \varphi \in U^{[u+2]}$. Since $\mathscr{D} \varphi \in U^{[u+2]}$, we may express

$$
\mathscr{D} \varphi=\sum_{\ell=0}^{\infty} \Delta^{\ell} \psi_{\ell} \quad\left(\psi_{\ell} \in \mathfrak{S}^{[u+2+\ell]}\right) .
$$

Putting

$$
\psi=\sum_{\ell \neq n} \frac{1}{(\ell+1)(\ell-n)} \Delta^{\ell+1} \psi_{\ell}=\sum_{\ell \neq n} \frac{1}{(\ell+1)(u+\ell+2)} \Delta^{\ell+1} \psi_{\ell},
$$

by virtue of (1.3) we have

$$
\begin{aligned}
\mathscr{D}(\varphi-\psi) & =\sum_{\ell} \Delta^{\ell} \psi_{\ell}-\sum_{\ell \neq n} \frac{(\ell+1)(u+2+2 \ell-\ell-1+1)}{(\ell+1)(u+\ell+2)} \Delta^{\ell} \psi_{\ell} \\
& =\Delta^{n} \psi_{n} .
\end{aligned}
$$

By virtue of Condition (C) there exists no isobaric element of index $n$ for $n>0$. If $n=0$, then $\mathfrak{S}^{[n]}=K$. Since $\mathscr{D} \varphi$ has no constant term, hence whenever $\mathscr{D}(\varphi-\psi)=\Delta^{n} \psi_{n}=0$. This shows that

$$
\begin{aligned}
& \varphi=\psi+(\varphi-\psi) \\
& \varphi-\psi \in \mathfrak{S}^{[u]}, \quad \phi \in U^{[u]},
\end{aligned}
$$

and thus $\varphi \in U^{[u]}$.

TheOREM 1.2 (Gram's theorem). If $w=\left(w_{1}, \cdots, w_{N}\right)$ satisfies Condition (C), then the following three conditions on an ideal a of $K\left[\xi_{1}, \cdots, \xi_{N}\right]$ are equivalent;

i) $\mathfrak{a}$ is an $\operatorname{sl}(2, K)$-admissible ideal.

ii) There exists a set of index-homogeneous binary semi-invariants $\left\{\varphi_{\lambda} \mid \lambda \in \Lambda\right\}$ such that $a$ is generated by $\left\{\Delta^{\ell} \varphi_{\lambda} \mid \ell=0,1,2, \cdots ; \lambda \in \Lambda\right\}$ as an ideal. 
Proof. Let $a$ be an $s l(2, K)$-admissible ideal. Then $a$ is an $s l(2, K)$ invariant submodule, hence by virtue of Theorem 1.1 we have

$$
\mathfrak{a}=\bigoplus_{\ell=0}^{\infty} \Delta^{\ell}(\mathfrak{a} \cap \mathfrak{S})=\bigoplus_{u} \bigoplus_{\ell=0}^{\infty} \Delta^{\ell}\left(\mathfrak{a} \cap \mathfrak{S}^{[u]}\right) \text {. }
$$

This shows that i) implies ii). Let $\left\{\varphi_{\lambda} \mid \lambda \in \Lambda\right\}$ be a set of index-homogeneous elements of $\mathfrak{S}$ such that $\mathfrak{a}$ is generated by $\left\{\Delta^{\ell} \varphi_{\lambda} \mid \ell=0,1,2, \cdots ; \lambda \in \Lambda\right\}$ as an ideal. Then elements of $\mathfrak{a}$ are linear combinations of

$$
\phi \Delta^{\ell} \varphi_{\lambda}\left(\phi \in K\left[\xi_{1}, \cdots, \xi_{N}\right]^{[u]}, \ell=0,1,2, \cdots ; \lambda \in \Lambda\right) .
$$

Let us examine the actions of $\mathscr{H}, \mathscr{D}, \Delta$ on such a element $\phi \Delta^{\ell} \varphi_{\lambda}$;

$$
\begin{aligned}
\mathscr{H}\left(\phi \Delta^{\ell} \varphi_{\lambda}\right) & =\mathscr{H} \phi \Delta^{\ell} \varphi_{\lambda}+\phi \mathscr{H} \Delta^{\ell} \varphi_{\lambda} \\
& =\left(\operatorname{index}(\phi)+\operatorname{index}\left(\varphi_{\lambda}\right)-2 \ell\right) \phi \Delta^{\ell} \varphi^{\lambda}, \\
\mathscr{D}\left(\phi \Delta^{\ell} \varphi_{\lambda}\right) & =\mathscr{D} \phi \Delta^{\ell} \varphi_{\lambda}+\ell\left(\operatorname{index}\left(\varphi_{\lambda}\right)-\ell+1\right) \phi \Delta^{\ell-1} \varphi_{\lambda}, \\
\Delta\left(\phi \Delta^{\ell} \varphi_{\lambda}\right) & =\Delta \phi \Delta^{\ell} \varphi_{\lambda}+\phi \Delta^{\ell+1} \varphi_{\lambda} .
\end{aligned}
$$

This shows that

$$
\mathscr{H} \mathfrak{a}, \quad \mathscr{D} \mathfrak{a}, \quad \Delta \mathfrak{a} \subset \mathfrak{a},
$$

namely $\mathfrak{a}$ is an $\operatorname{sl}(2, K)$-admissible ideal.

\section{§3. Robert's theorem}

3.1. We shall define the action of $G L(2, C)$ on the basic formal power series $f_{1}\left(\xi_{1} \mid z\right), \cdots, f_{N}\left(\xi_{N} \mid z\right)$ and shall prove Robert's theorem which gives a canonical isomorphisms between the algebra of semi-invariants $\mathbb{S}$ and the algebra of covariants.

For a complex number $u$ we mean by $z^{u}$ the convergent power series

$$
\sum_{\ell=0}^{\infty}\left(\begin{array}{l}
u \\
\ell
\end{array}\right)(z-1)^{\ell}
$$

in the disc $\{|z||z-1|<1\}$ with center at 1 , which is a branch of the many valued function $z^{u}$. follows:

Actions of elements $\left(\begin{array}{ll}\delta & \beta \\ \gamma & \alpha\end{array}\right)$ in $G L(2, C)$ satisfying $\delta \neq 0$ are defined as

$$
f_{j}\left(\left(\begin{array}{ll}
\delta & \beta \\
\gamma & \alpha
\end{array}\right) \xi_{j} \mid z\right)=(\gamma z+\delta)^{w_{j}} f_{j}\left(\xi_{j} \mid \frac{\alpha z+\beta}{\gamma z+\delta}\right) \quad(1 \leq j \leq N)
$$

namely 


$$
\begin{aligned}
& \sum_{\ell=0}^{\infty}\left(\begin{array}{c}
w_{j} \\
\ell
\end{array}\right)\left(\left(\begin{array}{ll}
\delta & \beta \\
\gamma & \alpha
\end{array}\right) \xi_{j}\right)^{(\ell)} z^{\ell} \\
&=(\gamma z+\delta)^{w_{j}} \sum_{\ell=0}^{\infty}\left(\begin{array}{c}
w_{j} \\
\ell
\end{array}\right) \xi_{j}^{(\ell)}\left(\frac{\alpha z+\beta}{\gamma z+\delta}\right)^{\ell} \\
&\left(\left(\begin{array}{cc}
\delta & \beta \\
\gamma & \alpha
\end{array}\right) \in G L(2, C), \delta \neq 0,1 \leq j \leq N\right) .
\end{aligned}
$$

We shall calculate $\left(\left(\begin{array}{ll}\delta & \beta \\ \gamma & \alpha\end{array}\right) \xi_{j}\right)^{(\ell)}$ explicitely.

Proposition 1.5.

$$
\left(\left(\begin{array}{ll}
\delta & \beta \\
\gamma & \alpha
\end{array}\right) \xi_{j}\right)^{(\ell)}=\sum_{p=0}^{\ell} \sum_{q=0}^{\infty}\left(\begin{array}{l}
\ell \\
p
\end{array}\right)\left(\begin{array}{c}
w_{j}-\ell \\
q
\end{array}\right) \xi_{j}^{(\ell-p+q)} \alpha^{\ell-p} \beta^{q} \gamma^{p} \delta^{w_{j}-\ell-q} .
$$

Proof. From (1.18) it follows

$$
\begin{aligned}
\left(\left(\begin{array}{ll}
\delta & \beta \\
\gamma & \alpha
\end{array}\right) \xi_{j}\right)^{(\ell)}= & \left.\left(\begin{array}{c}
w_{j} \\
\ell
\end{array}\right)^{-1} \frac{1}{\ell !}\left(\begin{array}{c}
d \\
d z
\end{array}\right)^{\ell}\left(\sum_{h=0}^{\infty}\left(\begin{array}{c}
w_{j} \\
h
\end{array}\right)\left(\left(\begin{array}{ll}
\delta & \beta \\
\gamma & \alpha
\end{array}\right) \xi_{j}\right)^{(h)} z^{h}\right)\right|_{z=0} \\
= & \left.\frac{1}{\ell !}\left(\begin{array}{c}
w_{j} \\
\ell
\end{array}\right)^{-1} \sum_{h=0}^{\infty}\left(\begin{array}{c}
w_{j} \\
h
\end{array}\right) \xi_{j}^{(h)}\left(\begin{array}{c}
d \\
d z
\end{array}\right)^{\ell}\left((\gamma z+\delta)^{w_{j}-h}(\alpha z+\beta)^{h}\right)\right|_{z=0} \\
= & \left.\left(\begin{array}{c}
w_{j} \\
\ell
\end{array}\right)^{-1} \sum_{h=0}^{\infty} \sum_{p=0}^{\ell}\left(\begin{array}{c}
w_{j} \\
h
\end{array}\right) \xi_{j}^{(h)} \frac{1}{(\ell-p) !}\left(\frac{d}{d z}\right)^{\ell-p}(\gamma z+\delta)^{w_{j}-h}\right|_{z=0} \\
& \times\left.\frac{1}{p !}\left(\frac{d}{d z}\right)^{p}(\alpha z+\beta)^{h}\right|_{z=0} \\
= & \left(\begin{array}{c}
w_{j} \\
\ell
\end{array}\right)^{-1} \sum_{h=0}^{\infty} \sum_{p=0}^{\ell}\left(\begin{array}{c}
w_{j} \\
h
\end{array}\right) \xi_{j}^{(h)}\left(\begin{array}{c}
h \\
p
\end{array}\right)\left(\begin{array}{c}
w_{j}-h \\
\ell_{-p}
\end{array}\right) \alpha^{p} \beta^{h-p} \gamma^{\ell-p} \delta^{w_{j}}
\end{aligned}
$$

Putting $h=p+q$ and replacing $p$ by $\ell-p$, we have

$$
\left(\left(\begin{array}{ll}
\delta & \beta \\
\gamma & \alpha
\end{array}\right) \xi_{j}\right)^{(\ell)}=\sum_{p=0}^{\ell} \sum_{q=0}^{\infty}\left(\begin{array}{c}
\ell \\
p
\end{array}\right)\left(\begin{array}{c}
w_{j}-\ell \\
q
\end{array}\right) \xi_{j}^{(\ell-\ell+q)} \alpha^{\ell-p} \beta^{q} \gamma^{p} \delta^{w_{j}-\ell-q}
$$

because

$$
\left(\begin{array}{c}
w_{j} \\
\ell
\end{array}\right)^{-1}\left(\begin{array}{c}
w_{j} \\
\ell+q
\end{array}\right)\left(\begin{array}{c}
w_{j}-p-q \\
\ell-p
\end{array}\right)\left(\begin{array}{c}
p+q \\
p
\end{array}\right)=\left(\begin{array}{c}
w_{j}-\ell \\
q
\end{array}\right)\left(\begin{array}{l}
\ell \\
p
\end{array}\right)
$$

Applying (1.19) to special type of elements, we have,

\section{Corollary 1.}

$$
\left(\left(\begin{array}{ll}
\alpha & 0 \\
0 & \alpha
\end{array}\right) \xi_{j}\right)^{(\ell)}=\alpha^{w_{j} \xi_{j}^{(\ell)}}
$$




$$
\begin{aligned}
& \left(\left(\begin{array}{ll}
1 & 0 \\
0 & \alpha
\end{array}\right) \xi_{j}\right)^{(\ell)}=\alpha^{\ell} \xi_{j}^{(\ell)}, \\
& \left(\left(\begin{array}{ll}
1 & 0 \\
t & 1
\end{array}\right) \xi_{j}\right)^{(\ell)}=\sum_{p=0}^{\ell}\left(\begin{array}{l}
\ell \\
p
\end{array}\right) \xi_{j}^{(\ell-p)} t^{p}, \\
& \left(\left(\begin{array}{ll}
1 & t \\
0 & 1
\end{array}\right) \xi_{j}\right)^{(\ell)}=\sum_{q=0}^{\infty}\left(\begin{array}{c}
w_{j}-\ell \\
q
\end{array}\right) \xi_{j}^{(\ell+q)} t^{q} .
\end{aligned}
$$

Corollary 2.

$$
\begin{gathered}
\mathscr{D} \varphi(\xi)=\left.\frac{d}{d t} \varphi\left(\left(\begin{array}{ll}
1 & 0 \\
t & 0
\end{array}\right) \xi\right)\right|_{t=0}, \\
\Delta \varphi(\xi)=\left.\frac{d}{d t} \varphi\left(\left(\begin{array}{ll}
1 & t \\
0 & 1
\end{array}\right) \xi\right)\right|_{t=0},
\end{gathered}
$$

Proof. Since both sides of (1.24), (1.25) are derivations acting on $\varphi(\xi)$, it is sufficient to prove (1.24), (1.25) for $\varphi(\xi)=\xi^{(\ell)}$. For such a case (1.22), (1.23) imply (1.24), (1.25), respectively.

3.2. Let us give the definition of covariants.

Definition 1.3. A covariant of index $u$ is a formal power series

$$
F\left(\xi_{1}, \cdots, \xi_{N} ; z\right)=\sum_{\ell=0}^{\infty}\left(\begin{array}{l}
u \\
\ell
\end{array}\right) c_{\ell}(\xi) z^{\ell}
$$

with coefficients $c_{\ell}(\xi)$ in $C\left[\xi_{1}, \cdots, \xi_{N}\right]$ such that

$$
\begin{aligned}
& F\left(\left(\begin{array}{ll}
\delta & \beta \\
\gamma & \alpha
\end{array}\right) \xi_{1}, \cdots,\left(\begin{array}{ll}
\delta & \beta \\
\gamma & \alpha
\end{array}\right) \xi_{N} ; z\right) \\
& \quad=(\gamma z+\delta)^{u} F\left(\xi_{1}, \cdots, \xi_{N} ; \frac{\alpha z+\beta}{\gamma z+\delta}\right) \quad\left(\left(\begin{array}{ll}
\delta & \beta \\
\gamma & \alpha
\end{array}\right) \in S L(2, C), \delta \neq 0\right) .
\end{aligned}
$$

We denote by $\mathscr{C}^{[u]}$ the vector space of covariants of index $u$, and denote $\mathscr{C}=\oplus_{u} \mathscr{C}^{[u]}$ is called the algebra of covariants.

LEMMA 1.9. If $F\left(\xi_{1}, \cdots, \xi_{N} ; z\right)$ is a covariant of index $u$, then $F\left(\xi_{1}, \cdots\right.$, $\left.\xi_{N} ; 0\right)$ is a binary semi-invariant of index $u$, and

$$
F\left(\xi_{1}, \cdots, \xi_{N} ; z\right)=\exp (z \Delta) F\left(\xi_{1}, \cdots, \xi_{N} ; 0\right) .
$$

Proof. We shall first prove (1.27). Applying $\left(\begin{array}{ll}1 & t \\ 0 & 1\end{array}\right)$ to $F\left(\xi_{1}, \cdots, \xi_{n} ; z\right)$, we have 


$$
\begin{aligned}
\Delta F\left(\xi_{1}, \cdots, \xi_{n} ; z\right) & =\left.\frac{d}{d t} F\left(\left(\begin{array}{ll}
1 & t \\
0 & 1
\end{array}\right) \xi_{1}, \cdots,\left(\begin{array}{ll}
1 & t \\
0 & 1
\end{array}\right) \xi_{n} ; z\right)\right|_{t=0} \\
& =\frac{d}{d t} F\left(\xi_{1}, \cdots, \xi_{n} ; z+t\right)=\frac{d}{d z} F\left(\xi_{1}, \cdots, \xi_{n} ; z\right)
\end{aligned}
$$

Since

$$
F\left(\xi_{1}, \cdots, \xi_{N} ; z\right)=\sum_{\ell=0}^{\infty} \frac{1}{\ell !}\left(\frac{d}{d z}\right)^{\ell} F\left(\xi_{1}, \cdots, \xi_{n} ; 0\right) z^{\ell}
$$

we have

$$
\begin{aligned}
F\left(\xi_{1}, \cdots, \xi_{N} ; z\right) & =\sum_{\ell=0}^{\infty} \frac{1}{\ell !} \Delta^{\ell} F\left(\xi_{1}, \cdots, \xi_{n} ; 0\right) z^{\ell} \\
& =\exp (z \Delta) F\left(\xi_{1}, \cdots, \xi_{N} ; 0\right)
\end{aligned}
$$

Similarly we have

$$
\begin{aligned}
\mathscr{D} F\left(\xi_{1}, \cdots, \xi_{n} ; 0\right) & =\left.\frac{d}{d t} F\left(\left(\begin{array}{cc}
1 & 0 \\
t & 1
\end{array}\right) \xi_{1}, \cdots,\left(\begin{array}{cc}
1 & 1 \\
t & 0
\end{array}\right) \xi_{N} ; z\right)\right|_{z=0, i=0} \\
& =\left.\frac{d}{d t}\left((t z+1)^{u} F\left(\xi_{1}, \cdots, \xi_{N} ; \frac{z}{t z+1}\right)\right)\right|_{z=0, t=0} \\
& =\left.\frac{d}{d t} F\left(\xi_{1}, \cdots, \xi_{N} ; 0\right)\right|_{t=0} \\
& =0 .
\end{aligned}
$$

This means that $F\left(\xi_{1}, \cdots, \xi_{N} ; 0\right)$ is a binary semi-invariant. By virtue of (1.20), (1.21).

$$
\begin{aligned}
& \left.\frac{d}{d t}\left(\left(\begin{array}{cc}
1+t & 0 \\
0 & (1+t)^{-1}
\end{array}\right) \xi_{j}\right)^{(\ell)}\right|_{t=0}=\left.\frac{d}{d t}\left((1+t)^{w_{j}-2 \ell} \xi_{j}^{(\ell)}\right)\right|_{t=0} \\
& \quad=\left(w_{j}-2 \ell\right) \xi_{j}^{(\ell)}=H \xi_{j}^{(\ell)} .
\end{aligned}
$$

Hence, to prove index $\left(F\left(\xi_{1}, \cdots, \xi ; 0\right)=u\right.$, it is sufficient to show

$$
\begin{aligned}
& \left.\frac{d}{d t} F\left(\left(\begin{array}{cc}
1+t & 0 \\
0 & (1+t)^{-1}
\end{array}\right) \xi_{1}, \cdots,\left(\begin{array}{cc}
1+t & 0 \\
0 & (1+t)^{-1}
\end{array}\right) \xi_{N} ; 0\right)\right|_{t=0} \\
& \quad=u F\left(\xi_{1}, \cdots, \xi_{N} ; 0\right) .
\end{aligned}
$$

Actually we have

$$
\left.\frac{d}{d t} F\left(\left(\begin{array}{cc}
1+t & 0 \\
0 & (1+t)^{-1}
\end{array}\right) \xi_{1}, \cdots,\left(\begin{array}{cc}
1+t & 0 \\
0 & (1+t)^{-1}
\end{array}\right) \xi_{N} ; 0\right)\right|_{t=0}
$$




$$
\begin{aligned}
& =\left.\frac{d}{d t}(1+t)^{u} F\left(\xi_{1}, \cdots, \xi_{N} ; \frac{z}{(1+t)^{2}}\right)\right|_{z=0, t=0} \\
& =u F\left(\xi_{1}, \cdots, \xi_{N} ; 0\right) .
\end{aligned}
$$

LEMmA 1.10. Let $\varphi\left(\xi_{1}, \cdots, \xi_{N}\right)$ be an element of $C\left[\xi_{1}, \cdots, \xi_{N}\right]$. Then we have

$$
\varphi\left(\left(\begin{array}{ll}
1 & z \\
0 & 1
\end{array}\right) \xi_{1}, \cdots,\left(\begin{array}{ll}
1 & z \\
0 & 1
\end{array}\right) \xi_{N}\right) \Phi_{w}(\varphi)
$$

where $\Phi_{w}$ is the isomorphism of $C\left[\xi_{1}, \cdots, \xi_{N}\right]$ such that

$$
\xi_{j}^{(\ell)} \longrightarrow \frac{1}{w_{j}\left(w_{j}-1\right) \cdots\left(w_{j}-\ell+1\right)} f_{j}^{(\ell)}\left(\xi_{j} \mid z\right) \text {. }
$$

Proof. Since

$$
f_{j}\left(\xi_{j} \mid z\right)=\sum_{\ell=0}^{\infty}\left(\begin{array}{c}
w_{j} \\
\ell
\end{array}\right) \xi_{j}^{(\ell)} z^{\ell}
$$

by virtue of (1.23) we have

$$
\begin{aligned}
& \Phi_{w}\left(\xi_{j}^{(\ell)}\right)=\frac{1}{w_{j}\left(w_{j}-1\right) \cdots\left(w_{j}-\ell+1\right)} f_{j}^{(\ell)}\left(\xi_{j} \mid z\right) \\
& =\frac{1}{w_{j}\left(w_{j}-1\right) \cdots\left(w_{j}-\ell+1\right)} \sum_{q=0}^{\infty}\left(\begin{array}{c}
w_{j} \\
\ell+q
\end{array}\right) \xi_{j}^{(\ell+q)}\left(\frac{d}{d z}\right)^{\ell} z^{\ell+q} \\
& \quad=\sum_{q=0}^{\infty} \frac{w_{j}\left(w_{j}-1\right) \cdots\left(w_{j}-\ell-q+1\right)}{w_{j}\left(w_{j}-1\right) \cdots\left(w_{j}-\ell+1\right)} \frac{(\ell+q) !}{(\ell+q-\ell) !} \xi_{j}^{(\ell+q)} z^{q} \\
& \quad=\sum_{q=0}^{\infty}\left(\begin{array}{c}
w_{j}-\ell \\
q
\end{array}\right) \xi_{j}^{(\ell+q)} z^{q}=\left(\left(\begin{array}{ll}
1 & z \\
0 & 1
\end{array}\right) \xi_{j}\right)^{(\ell)} .
\end{aligned}
$$

Since $\Phi_{w}$ is an algebra isomorphism, we can conclude

$$
\varphi\left(\left(\begin{array}{ll}
1 & z \\
0 & 1
\end{array}\right) \xi_{1}, \cdots,\left(\begin{array}{ll}
1 & z \\
0 & 1
\end{array}\right) \xi_{N}\right)=\Phi_{w}(\varphi)
$$

LEMma 1.11. An element $\varphi\left(\xi_{1}, \cdots, \xi_{N}\right)$ of $C\left[\xi_{1}, \cdots, \xi_{N}\right]$ is a binary semiinvariant of index $u$, if and only if

$$
\varphi\left(\left(\begin{array}{cc}
\delta & 0 \\
\gamma & \delta^{-1}
\end{array}\right) \xi_{1}, \cdots,\left(\begin{array}{cc}
\delta & 0 \\
\gamma & \delta^{-1}
\end{array}\right) \xi_{N}\right)=\delta^{u} \varphi\left(\xi_{1}, \cdots, \xi_{N}\right) \quad(\gamma, \delta \in C, \delta \neq 0)
$$

Proof. Let $\varphi\left(\xi_{1}, \cdots, \xi_{N}\right)$ be an element $K\left[\xi_{1}, \cdots, \xi_{N}\right]^{[u]}$. Since

$$
\left(\left(\begin{array}{cc}
\delta & 0 \\
0 & \delta^{-1}
\end{array}\right) \xi_{j}\right)^{(\ell)}=\delta^{w_{j-2 \ell} \xi_{j}^{(\ell)}}
$$


We have

$$
\left.\varphi\left(\begin{array}{cc}
\delta & 0 \\
0 & \delta^{-1}
\end{array}\right) \xi_{1}, \cdots,\left(\begin{array}{cc}
\delta & 0 \\
0 & \delta^{-1}
\end{array}\right) \xi_{N}\right)=\delta^{u} \varphi\left(\xi_{1}, \cdots, \xi_{N}\right)
$$

Therefore it is enough to show $\mathscr{D} \varphi=0$ if and only if

$$
\varphi\left(\left(\begin{array}{ll}
1 & 0 \\
t & 1
\end{array}\right) \xi_{1}, \cdots,\left(\begin{array}{ll}
1 & 0 \\
t & 1
\end{array}\right) \xi_{N}\right)=\varphi\left(\xi_{1}, \cdots, \xi_{N}\right) \quad(t \in C) .
$$

Since

$$
\mathscr{D} \varphi\left(\left(\xi_{1}, \cdots, \xi_{N}\right)=\frac{d}{d t} \varphi\left(\left(\begin{array}{ll}
1 & 0 \\
t & 1
\end{array}\right) \xi_{1}, \cdots,\left(\begin{array}{ll}
1 & 0 \\
t & 1
\end{array}\right) \xi_{N}\right)\right.
$$

the "if" part is obvious. Assume $\mathscr{D} \varphi=0$. Then we have

$$
\begin{aligned}
& \left.\frac{d}{d t} \varphi\left(\left(\begin{array}{ll}
1 & 0 \\
t & 1
\end{array}\right) \xi_{1}, \cdots,\left(\begin{array}{ll}
1 & 1 \\
t & 0
\end{array}\right) \xi_{N}\right)\right|_{t=s} \\
& \quad=\frac{d}{d t} \varphi\left(\left(\begin{array}{cc}
1 & 0 \\
t+s & 1
\end{array}\right) \xi_{1}, \cdots,\left(\begin{array}{ll}
1 & 0 \\
t+s & 1
\end{array}\right) \xi_{N}\right)_{t=0} \\
& \quad=\left.\frac{d}{d t}\left(\left(\begin{array}{ll}
1 & 0 \\
t & 1
\end{array}\right)\left(\begin{array}{ll}
1 & 0 \\
0 & s
\end{array}\right) \xi_{1}, \cdots,\left(\begin{array}{ll}
1 & 1 \\
0 & t
\end{array}\right)\left(\begin{array}{ll}
1 & 0 \\
s & 1
\end{array}\right) \xi_{N}\right)\right|_{t=0} \\
& \quad=\mathscr{D} \varphi\left(\left(\begin{array}{ll}
1 & 0 \\
s & 1
\end{array}\right) \xi_{1}, \cdots,\left(\begin{array}{ll}
1 & 0 \\
s & 0
\end{array}\right) \xi_{N}\right)=0 .
\end{aligned}
$$

This shows that

$$
\varphi\left(\left(\begin{array}{ll}
1 & 0 \\
t & 1
\end{array}\right) \xi_{1}, \cdots,\left(\begin{array}{ll}
1 & 0 \\
t & 1
\end{array}\right) \xi_{N}\right)=\varphi\left(\xi_{1}, \cdots, \xi_{N}\right) .
$$

Let us sum up the above results in the next theorem which is the analogy of Robert's theorem in classical invariant theory.

TheOREM 1.3 (Robert's theorem). The isomorphism $\Phi_{w}$ gives an $\boldsymbol{C}$ algebra isomorphism of the algebra of binary semi-invariants $\mathbb{S}$ onto the algebra of covariants $\mathscr{C}$. Moreover

$$
\Phi_{w}\left(\Im^{[u]}\right)=\mathscr{C}^{[u]} .
$$

Proof. Let $\varphi\left(\xi_{1}, \cdots, \xi_{N}\right)$ be an element of $\mathfrak{S}^{[u]}$. Using the matric relation

$$
\left(\begin{array}{ll}
1 & z \\
0 & 1
\end{array}\right)\left(\begin{array}{ll}
\delta & \beta \\
\gamma & \alpha
\end{array}\right)=\left(\begin{array}{cc}
\gamma z+\delta & 0 \\
\gamma & (\gamma z+\delta)^{-1}
\end{array}\right)\left(\begin{array}{cc}
1 & (\alpha z+\beta) /(\gamma z+\delta) \\
0 & 1
\end{array}\right) \quad\left(\left(\begin{array}{ll}
\delta & \beta \\
\gamma & \alpha
\end{array}\right) \in S L(2, C)\right),
$$


by virtue of (1.20), (1.21), (1.28), (1.29) we have

$$
\begin{aligned}
& \Phi_{w}(\varphi)\left(\left(\begin{array}{ll}
\delta & \beta \\
\gamma & \alpha
\end{array}\right) \xi_{1}, \cdots,\left(\begin{array}{ll}
\delta & \beta \\
\gamma & \alpha
\end{array}\right) \xi_{N} ; z\right) \\
& =\varphi\left(\left(\begin{array}{ll}
1 & z \\
0 & 1
\end{array}\right)\left(\begin{array}{ll}
\delta & \beta \\
\gamma & \alpha
\end{array}\right) \xi_{1}, \cdots,\left(\begin{array}{ll}
1 & z \\
0 & 1
\end{array}\right)\left(\begin{array}{ll}
\delta & \beta \\
\gamma & \alpha
\end{array}\right) \xi_{N}\right) \\
& =\varphi\left(\left(\begin{array}{cc}
\gamma z+\delta & 0 \\
\gamma & (\gamma z+\delta)^{-1}
\end{array}\right)\left(\begin{array}{cc}
1 & (\alpha z+\beta) /(\gamma z+\delta) \\
0 & 1
\end{array}\right) \xi_{1}, \cdots,\right. \\
& \left.\left(\begin{array}{cc}
\gamma z+\delta & 0 \\
\gamma & (\gamma z+\delta)^{-1}
\end{array}\right)\left(\begin{array}{cc}
1 & (\alpha z+\beta) /(\gamma z+\delta) \\
0 & 1
\end{array}\right) \xi_{N}\right) \\
& =(\gamma z+\delta)^{u} \varphi\left(\left(\begin{array}{cc}
1 & (\alpha z+\beta) /(\gamma z+\delta) \\
0 & 1
\end{array}\right) \xi_{1}, \cdots,\left(\begin{array}{cc}
1 & (\alpha z+\beta) /(\gamma z+\gamma) \\
0 & 1
\end{array}\right) \xi_{N}\right) \\
& =(\gamma z+\delta)^{u} \Phi_{w}(\varphi)\left(\xi_{1}, \cdots, \xi_{N} ; \frac{\alpha z+\beta}{\gamma z+\delta}\right) .
\end{aligned}
$$

This means that $\Phi_{w}(\varphi) \in \mathscr{C}^{[u]}$. Conversely, if $F\left(\xi_{1}, \cdots, \xi_{N} ; z\right)$ is an element of $\mathscr{C}^{[u]}$, then by virtue of Lemmas 1.7 and 1.9 we have

$$
F\left(\xi_{1}, \cdots, \xi_{N} ; z\right)=\exp (z \Delta) F\left(\xi_{1}, \cdots, \xi_{N} ; 0\right)=\Phi_{w}(\varphi)\left(\xi_{1}, \cdots, \xi_{N} ; z\right) .
$$

\section{§3. Binary semi-invariants and automorphic forms}

3.1. Rankin is the first who noticed the relation between binary semiinvariants and automorphic forms ${ }^{1}$. In this paragraph we shall give more complete and systematic treatment of the relation.

To aboid the confusion between definitions in invariant theory and automorphic function theory, we shall use the following language:

Automorphic form of index $-2 k$, instead of automorphic form of dimension $-2 k$.

LEMMA 1.12.

$$
\begin{array}{r}
\frac{1}{w(w-1) \cdots(w-\ell+1)}\left(\frac{d}{d((\alpha z+\beta) /(\gamma z+\delta))}\right)^{\ell}\left((\gamma z+\delta)^{-w} h(z)\right) \\
=\sum_{k=0}^{\ell}(-1)^{k}\left(\begin{array}{l}
\ell \\
k
\end{array}\right) \frac{(d / d z)^{\ell-k} h(z)}{w(w-1) \cdots(w-\ell+k+1)} \gamma^{k}(\gamma z+\delta)^{-w+2 \ell-k} \\
\left.\left(\begin{array}{ll}
\delta & \beta \\
\gamma & \alpha
\end{array}\right) \in S L(2, C)\right) .
\end{array}
$$

Proof. Let us prove (1.30) by induction on $\ell$. It is obvious for $\ell=0$.

1) See [2]. 
Assume (1.30) for $\ell$ and let us show (1.30) for $\ell+1$. Since $\alpha \delta-\beta \gamma=1$, we have

$$
\frac{d}{d((\alpha z+\beta) /(\gamma z+\delta))}=(\gamma z+\delta)^{2} \frac{d}{d z}
$$

and

$$
\begin{aligned}
& \frac{1}{w(w-1) \cdots(w-\ell)}\left(\frac{d}{d((\alpha z+\beta) /(\gamma z+\delta))}\right)^{\ell+1}\left((\gamma z+\delta)^{-w} h(z)\right) \\
& =\frac{1}{w-\ell}(\gamma z+\delta)^{2} \frac{d}{d z}\left\{\frac{1}{w(w-1) \cdots(w-\ell+1)}\right. \\
& \left.\times\left(\frac{d}{d((\alpha z+\beta) /(\gamma z+\delta))}\right)^{\ell}\left((\gamma z+\delta)^{-w} h(z)\right)\right\} \\
& =\frac{1}{w-\ell}(\gamma z+\delta)^{2} \frac{d}{d z}\left\{\sum_{k=0}^{\ell}(-1)^{k}\left(\begin{array}{l}
\ell \\
k
\end{array}\right) \frac{(d / d z)^{\ell-k} h(z)}{w(w-1) \cdots(w-\ell+k+1)}\right. \\
& \left.\times \gamma^{k}(\gamma z+\delta)^{-w+2 \ell-k}\right\} \\
& =\frac{1}{w-\ell} \sum_{k=0}^{\ell}(-1)^{k}\left(\begin{array}{l}
\ell \\
k
\end{array}\right) \frac{(d / d z)^{\ell+1-k} h(z)}{w(w-1) \cdots(w-\ell-1+k+1)} \\
& \times(w-\ell+k) \gamma^{k}(\gamma z+\delta)^{-w+2(\ell+1)-k} \\
& +\frac{1}{w-\ell} \sum_{k=0}^{\ell}(-1)^{k}\left(\begin{array}{l}
\ell \\
k
\end{array}\right) \frac{(d / d z)^{\ell+1-k-1} h(z)}{w(w-1) \cdots(w-\ell-1+k+1+1)} \\
& \times(-w+2 \ell-k) \gamma^{k+1}(\gamma z+\delta)^{-w+2(\ell+1)-k-1} \\
& =\frac{1}{w(w-1) \cdots(w-\ell)}\left(\frac{d}{d z}\right)^{\ell+1}\left(h(z) \cdot(\gamma z+\delta)^{-w-2(\ell+1)}\right. \\
& \left.+(-1)^{\ell+1} \gamma^{\ell+1}(\gamma z+\delta)^{-w+\ell+1} h(z)\right) \\
& +\frac{1}{w-\ell} \sum_{k=1}^{\ell}(-1)^{k} \frac{(d / d z)^{\ell+1-k} h z}{w(w-1) \cdots(w-\ell-1+k+1)} \\
& \cdot \gamma^{k}(\gamma z+\delta)^{-w+2(\ell+1)-k}\left\{\left(\begin{array}{l}
\ell \\
k
\end{array}\right)(w-\ell+k)-\left(\begin{array}{c}
\ell \\
k-1
\end{array}\right)(-w+2 \ell-k+1)\right\} \text {. }
\end{aligned}
$$

Since

$$
\begin{aligned}
& \frac{1}{w-\ell}\left\{\left(\begin{array}{l}
\ell \\
k
\end{array}\right)(w-\ell+k)-\left(\begin{array}{c}
\ell \\
k-1
\end{array}\right)(-w+2 \ell-k+1)\right\} \\
& \quad=\frac{1}{w-\ell} \frac{\ell !}{k !(\ell-k+1) !}\{(w-\ell+k)(\ell-k+1)+(w-2 \ell+k-1) k\} \\
& \quad=\frac{1}{w-\ell} \frac{\ell !}{k !(\ell-k+1) !}(w \ell+w-\ell(\ell+1))=\left(\begin{array}{c}
\ell+1 \\
k
\end{array}\right),
\end{aligned}
$$


we have

$$
\begin{gathered}
\frac{1}{w(w-1) \cdots(w-\ell)}\left(\frac{d}{d((\alpha z+\beta) /(\gamma z+\delta))}\right)^{\ell+1}\left((\gamma z+\delta)^{-w} h(z)\right) \\
=\sum_{k=0}^{\ell+1}(-1)^{k}\left(\begin{array}{c}
\ell+1 \\
k
\end{array}\right) \frac{(d / d z)^{\ell+1-k} h(z)}{w(w-1) \cdots(w-\ell-1+k+1)} \\
\quad \times \gamma^{k}(\gamma z+\delta)^{-w+2(\ell+1)-k} .
\end{gathered}
$$

Let $D$ be a domain in $C$ containing the origin, and $\Gamma$ be a subgroup of $S L(2, C)$ which acts on $D$ properly discontinuously. We mean by a $\Gamma$ automorphic form of index $u$ a meromorphic function $F(z)$ on $D$ such that

$$
F\left(\frac{\alpha z+\beta}{\gamma z+\delta}\right)=(\gamma z+\delta)^{u} F(z) \quad\left(\left(\begin{array}{ll}
\delta & \beta \\
\gamma & \alpha
\end{array}\right) \in \Gamma\right)
$$

and $F(z)$ is also meromorphic at the cusps of $\Gamma$. A $\Gamma$-automorphic form is called to be integral, if it is holomorphic at each point in $D$ and each cusps.

Theorem 1.4. Let $k_{1}, \cdots, k_{N}$ be positive integers and put $w_{1}=-2 k_{1}, \cdots$, $w_{N}=-2 k_{N}$. Let $\varphi(\xi)$ be a binary semi-invariant of index $u$, where index is given by $w=\left(w_{1}, \cdots, w_{N}\right)$. Let $h_{1}(z), \cdots, h_{N}(z)$ be $\Gamma$-automorphic forms of index $-2 k_{1}, \cdots,-2 k_{N}$, respectively, and let $\Phi(\varphi)(z)$ be the differential polynomial given by the replacement;

$$
\begin{aligned}
\xi_{j}^{(\ell)} \longrightarrow \frac{1}{w_{j}\left(w_{j}-1\right) \cdots\left(w_{j}-\ell+1\right)} & \left(\frac{d}{d z}\right)^{\ell} h_{j}(z) \\
& (\ell=0,1,2, \cdots ; 1 \leq j \leq N)
\end{aligned}
$$

in $\varphi(\xi)$. Then $\Phi(\varphi)(z)$ is a $\Gamma$-automorphic form of index $u$. If $h_{1}(z), \cdots, h_{N}(z)$ are integral, then $\Phi(\varphi)(z)$ is also integral.

Proof. Since $\Phi(\varphi)(z)$ is a differential polynomial in $h_{1}(z), \cdots, h_{N}(z)$ with constant coefficients and $h_{1}(z), \cdots, h_{N}(z)$ are meromorphic in $D$ and at the cusps, hence $\Phi(\varphi)(z)$ has the same properties. By virtue of (1.30) for each $\left(\begin{array}{ll}\delta & \beta \\ \gamma & \alpha\end{array}\right)$ in $\Gamma$ we have

$$
\begin{aligned}
& \frac{1}{w_{j}\left(w_{j}-1\right) \cdots\left(w_{j}-\ell+1\right)}\left(\frac{d}{d((\alpha z+\beta) /(\gamma z+\delta))}\right)^{\ell} h_{j}\left(\frac{\alpha z+\beta}{\gamma z+\delta}\right) \\
& =\frac{1}{w_{j}\left(w_{j}-1\right) \cdots\left(w_{j}-\ell+1\right)}\left(\frac{d}{d((\alpha z+\beta) /(\gamma z+\delta))}\right)^{\ell}\left((\gamma z+\delta)^{-w_{j}} h_{j}(z)\right)
\end{aligned}
$$




$$
\begin{gathered}
=\sum_{k=0}^{\ell}(-1)^{k}\left(\begin{array}{l}
\ell \\
k
\end{array}\right) \frac{(d / d z)^{\ell-k} h_{j}(z)}{w_{j}\left(w_{j}-1\right) \cdots\left(w_{j}-\ell+k+1\right)} \gamma^{k}(\gamma z+\delta)^{-w_{j}+2 \ell-k} \\
=(\gamma z+\delta)^{-w_{j}+2 \ell} \sum_{k=0}^{\ell}\left(\begin{array}{l}
\ell \\
k
\end{array}\right) \frac{(d / d z)^{\ell-k}}{w_{j}\left(w_{j}-1\right) \cdots\left(w_{j}-\ell+k+1\right)} \\
\times\left(-\gamma(\gamma z+\delta)^{-1}\right)^{k} .
\end{gathered}
$$

Since $\varphi\left(\cdots, \xi_{j}^{(\ell)}, \cdots\right)$ is a binary semi-invariant of index $u$, putting $t=$ $-\gamma(\gamma z+\delta)^{-1}$, we have

$$
\begin{aligned}
& \Phi(\varphi)\left(\frac{\alpha z+\beta}{\gamma z+\delta}\right)=\varphi\left(\cdots,(\gamma z+\delta)^{-w_{j}+2 \ell} \sum_{k=0}^{\ell}\left(\begin{array}{l}
\ell \\
k
\end{array}\right)\right. \\
& =(\gamma z+\delta)^{u} \varphi\left(\cdots, \sum_{k=0}^{\ell}\left(\begin{array}{l}
\ell \\
k
\end{array}\right) \frac{(d / d z)^{\ell-k} h_{j}(z)}{w_{j}\left(w_{j}-1\right) \cdots\left(w_{j}-\ell+k+1\right)} t^{k}, \cdots\right) \\
& =\left.(\gamma z+\delta)^{n} \varphi\left(\left(\begin{array}{ll}
1 & 1 \\
t & 0
\end{array}\right) \xi_{1}, \cdots,\left(\begin{array}{ll}
1 & 0 \\
t & 1
\end{array}\right) \xi_{N}\right)\right|_{\xi_{j}^{(\ell)}}=\frac{(d / d z)^{\ell-k} h_{j}(z)}{w_{j}\left(w_{j}-1\right) \cdots\left(w_{j}-\ell+1\right)} \\
& =\left.(\gamma z+\delta)^{u} \varphi\left(\xi_{1}, \cdots, \xi_{N}\right)\right|_{\xi_{j}^{(\ell)}}=\frac{(d / d z)^{\ell} h_{j}(z)}{w_{j}\left(w_{j}-1\right) \cdots\left(w_{j}-\ell+1\right)} \\
& =(\gamma z+\delta)^{u} \Phi_{\varphi}(z) .
\end{aligned}
$$

Since $\Phi(\varphi)(z)$ is a differential polynomial in $h_{1}(z), \cdots, h_{N}(z), \Phi(\varphi)(z)$ is integral provided that $h_{1}(z), \cdots, h_{N}(z)$ are integral.

We shall show the converse of Theorem 1.4 is also true provided that the Zariski closure of $\Gamma$ is $S L(2)$.

Proposition 1.6. Let $h_{1}(z), \cdots, h_{N}(z)$ be $\Gamma$-automorphic forms of index $-2 k_{1}, \cdots,-2 k_{N}$, respectively, and let $a$ be the differential ideal of

$$
C\left[\left(\left(\frac{d}{d z}\right)^{\ell} y_{j}\right)_{\ell=0,1,2, \cdots, 1 \leq j \leq N}\right]
$$

consisting all the elements annihilated by $\left(h_{1}(z), \cdots, h_{N}(z)\right)$. Put $w_{1}=$ $-2 k_{1}, \cdots, w_{N}=-2 k_{N}$. If the Zariski closure of $\Gamma$ coincides with $S L(2)$, then $\mathfrak{a}$ is an sl(2)-admissible ideal with respect to $w=\left(w_{1}, \cdots, w_{N}\right)$.

Proof. Replacing

$$
\begin{gathered}
\left(z, y_{1}, \cdots, y_{N}\right) \longrightarrow\left(\left(\frac{\alpha z+\beta}{\gamma z+\delta}\right), h_{1}\left(\frac{\alpha z+\beta}{\delta z+\delta}\right), \cdots, h_{N}\left(\frac{\alpha z+\beta}{\gamma z+\delta}\right)\right) \\
\left(\left(\begin{array}{ll}
\delta & \beta \\
\gamma & \alpha
\end{array}\right) \in \Gamma\right)
\end{gathered}
$$


in an element

$$
F\left(\cdots, \frac{(d / d z)^{\ell} y_{j}}{w_{j}\left(w_{j}-1\right) \cdots\left(w_{j}-\ell+1\right)}, \cdots\right)
$$

in $a$, by virtue of (1.30) we have

$$
\begin{array}{rl}
0 & F\left(\cdots, \frac{1}{w_{j}\left(w_{j}-1\right) \cdots\left(w_{j}-\ell+1\right)}\right. \\
& \left.\quad \times\left(\frac{d}{d((\alpha z+\beta) /(\gamma z+\delta))}\right)^{\ell} h_{j}\left(\frac{\alpha z+\beta}{\gamma z+\delta}\right), \cdots\right) \\
=F\left(\cdots, \frac{1}{w_{j}\left(w_{j}-1\right) \cdots\left(w_{j}-\ell+1\right)}\right. \\
\left.\quad \times\left(\frac{d}{d((\alpha z+\beta) /(\gamma z+\delta))}\right)^{\ell}\left((\gamma z+\delta)^{-w_{j}} h_{j}(z)\right), \cdots\right) \\
=F\left(\cdots,(\gamma z+\delta)^{-w_{j}+2 \ell} \sum_{k=0}^{\ell}\left(\begin{array}{l}
\ell \\
k
\end{array}\right) \frac{(d / d z)^{\ell-k} h_{j}(z)}{w_{j}\left(w_{j}-1\right) \cdots\left(w_{j}-\ell+k+1\right)}\right. \\
\left.\quad \times\left(-\gamma(\gamma z+\delta)^{-1}\right)^{k}, \cdots\right) .
\end{array}
$$

Putting $s=\gamma z+\delta$ and $t=-\gamma(\gamma z+\delta)^{-1}$, we have

$$
F\left(\cdots, s^{2\left(k_{j}+\ell\right)} \sum_{k=0}^{\ell}\left(\begin{array}{l}
\ell \\
k
\end{array}\right) \frac{(d / d z)^{\ell-k} h_{j}(z)}{w_{j}\left(w_{j}-1\right) \cdots\left(w_{j}-\ell+k+1\right)} t^{k}, \cdots\right)=0 .
$$

This is an algebraic equation in $s$ and $t$. Since the Zariski closure of $\Gamma$ is $S L(2)$, we may put $s=1$ and consider $t$ a variable. Hence we have

$$
F\left(\cdots, \sum_{k=0}^{\ell}\left(\begin{array}{l}
\ell \\
k
\end{array}\right) \frac{t^{k}}{w_{j}\left(w_{j}-1\right) \cdots\left(w_{j}-\ell+k+1\right)}\left(\frac{d}{d z}\right)^{\ell-k} h_{j}(z), \cdots\right)=0 .
$$

Using the algebra isomorphism $\Theta_{w}$. we have

$$
\begin{aligned}
& F\left(\left(\begin{array}{ll}
1 & 0 \\
t & 1
\end{array}\right) \xi_{1}, \cdots,\left(\begin{array}{ll}
1 & 0 \\
t & 1
\end{array}\right) \xi_{N}\right) \\
& \quad=F\left(\cdots, \sum_{k=0}^{\ell}\left(\begin{array}{l}
\ell \\
k
\end{array}\right) \xi_{j}^{(\ell-k)} t^{k}, \cdots\right) \in \Theta_{u}^{-1}(\mathfrak{a}) .
\end{aligned}
$$

From the definition of $\mathscr{D}$

$$
\mathscr{D} F\left(\xi_{1}, \cdots, \xi_{N}\right)=\left.\frac{d}{d t} F\left(\left(\begin{array}{ll}
1 & 0 \\
t & 1
\end{array}\right) \xi_{1}, \cdots,\left(\begin{array}{ll}
1 & 0 \\
t & 1
\end{array}\right) \xi_{N}\right)\right|_{t=0} .
$$

This means that $\Theta_{w}(\mathscr{D} F)$ is the coefficient of $t$ in 


$$
F\left(\cdots, \sum_{k=0}^{\ell}\left(\begin{array}{l}
\ell \\
k
\end{array}\right) \frac{t^{k}}{w_{j}\left(w_{j}-1\right) \cdots\left(w_{j}-\ell+k+1\right)}\left(\frac{d}{d z}\right)^{\ell-k} y_{j} \cdots\right),
$$

and thus

$$
\left.\mathscr{D} F(\xi)\right|_{\xi_{j}^{(\ell)}}=\frac{(d / d z)^{\ell} h_{j}(z)}{w_{j}\left(w_{j}-1\right) \cdots\left(w_{j}-\ell+1\right)}=0 .
$$

Namely $\mathscr{D}\left(\Theta_{w}^{-1}(\mathfrak{a})\right) \subset \Theta_{w}^{-1}(\mathfrak{a})$. Since $d / d z \circ \Theta_{w}=\Theta_{w} \circ \Delta$, we have

$$
\Delta\left(\Theta_{w}^{-1}(\mathfrak{a})\right)=\Theta_{w}^{-1}\left(\frac{d}{d z} \mathfrak{a}\right) \subset \Theta_{w}^{-1}(\mathfrak{a}) .
$$

This shows that $\Theta_{w}^{-1}(\mathfrak{a})$ is an $\operatorname{sl}(2)$-admissible ideal and thus $\mathfrak{a}$ is $s l(2)$-admissible with respect to $w=\left(w_{1}, \cdots, w_{N}\right)$.

TheOREm 1.5. Let $k_{1}, \cdots, k_{N}$ be positive integers and put $w_{1}=-2 k_{1}, \cdots$, $w_{N}=-2 k_{N}$. Let $h_{1}(z), \cdots, h_{N}(z)$ be $\Gamma$-automorphic forms of index $-2 k_{1}, \cdots$, $-2 k_{N}$, which are holomorphic at the origin. If the Zariski closure of coincides with $S L(2)$, the algebra of covariants of $\left(h_{1}(z), \cdots, h_{N}(z)\right)$ with respect to $w=\left(w_{1}, \cdots, w_{N}\right)$ coincides with the algebra of $\Gamma$-automorphic forms which can be expressed as differential polynomials in $h_{1}(z), \cdots, h_{N}(z)$ with constant coefficients.

Proof. We assume that a differential polynomial with constant coefficients in $h_{1}(z), \cdots, h_{N}(z)$

$$
F\left(\cdots, \frac{(d / d z)^{\ell} h_{j}(z)}{w_{j}\left(w_{j}-1\right) \cdots\left(w_{j}-\ell+1\right)}, \cdots\right)
$$

is a non-constant $\Gamma$-automorphic form of index $u=-2 m$. Then for each $\left(\begin{array}{ll}\delta & \beta \\ \gamma & \alpha\end{array}\right) \in \Gamma$ we have

$$
\begin{aligned}
0= & F\left(\cdots, \frac{1}{w_{j}\left(w_{j}-1\right) \cdots\left(w_{j}-\ell+1\right)}\right. \\
& \left.\quad \times\left(\frac{d}{d((\alpha z+\beta) /(\gamma z+\delta)}\right)^{\ell} h_{j}\left(\frac{\alpha z+\beta}{\gamma z+\delta}\right), \cdots\right) \\
& -(\gamma z+\delta)^{2 m} F\left(\cdots, \frac{1}{w_{j}\left(w_{j}-1\right) \cdots\left(w_{j}-\ell+1\right)}\left(\frac{d}{d z}\right)^{\ell} h_{j}(z), \cdots\right) \\
= & F\left(\cdots,(\gamma z+\delta)^{2\left(k_{j}+\ell\right)} \sum_{k=1}^{\ell}\left(\begin{array}{l}
\ell \\
k
\end{array}\right) \frac{\left(-\gamma(\gamma z+\delta)^{-1}\right)^{k}}{w_{j}\left(w_{j}-1\right) \cdots\left(w_{j}-\ell+k+1\right)}\right. \\
& \left.\quad \times\left(\frac{d}{d z}\right)^{\ell-k} h_{j}(z), \cdots\right)
\end{aligned}
$$




$$
-(\gamma z+\delta)^{2 m} F\left(\cdots, \frac{1}{w_{j}\left(w_{j}-1\right) \cdots\left(w_{j}-\ell+1\right)}\left(\frac{d}{d z}\right)^{\ell} h_{j}(z), \cdots\right) .
$$

This is an algebraic equation in $s=\gamma z+\delta$ and $t=-\gamma(\gamma z+\delta)^{-1}$. Since the Zariski closure of $\Gamma$ is $S L(2)$. we may regard $s$ and $t$ as independent variables. Let $F(\xi)=\sum_{\ell} F^{[-2 \ell]}(\xi)$ be the index-homogeneous decomposition of $F(\xi)$. Let $\mathfrak{a}$ be the ideal consisting of all the polynomials $\varphi\left(\cdots, \xi_{j}^{(\ell)}, \cdots\right)$ such that

$$
\varphi\left(\cdots, \frac{(d / d z)^{\ell} h_{j}(z)}{w_{j}\left(w_{j}-1\right) \cdots\left(w_{j}-\ell+1\right)}, \cdots\right)=0 .
$$

Then $\mathfrak{a}$ is an $\operatorname{sl}(2)$-admissible ideal. We shall show that $\sum_{\ell \neq m} F^{[-2 \ell]}(\xi) \in \mathfrak{a}$. Putting $t=0$, we have

$$
\begin{aligned}
& F\left(\cdots, s^{2\left(k_{j}+\ell\right)} \frac{(d / d z)^{\ell} h_{j}(z)}{w_{j}\left(w_{j}-1\right) \cdots\left(w_{j}-\ell+1\right)}, \cdots\right) \\
& \quad=s^{2 m} F\left(\cdots, \frac{(d / d z)^{\ell} h_{j}(z)}{w_{j}\left(w_{j}-1\right) \cdots\left(w_{j}-\ell+1\right)}, \cdots\right) .
\end{aligned}
$$

Namely

$$
F\left(\cdots, s^{-w_{j}+2 \ell} \xi_{j}^{(\ell)}, \cdots\right)-s^{2 m} F\left(\cdots, \xi_{j}^{(\ell)}, \cdots\right) \in \mathfrak{a},
$$

and thus

$$
\begin{gathered}
\sum_{\ell} s^{2 \ell} F^{[-2 \ell]}\left(\cdots, \xi_{j}^{(\ell)}, \cdots\right)-s^{2 m} F\left(\cdots, \xi_{j}^{(\ell)}, \cdots\right) \\
=s^{2 m} \sum_{\ell \neq m} s^{2(\ell-m)} F^{[-2 \ell]}\left(\cdots, \xi_{j}^{(\ell)}, \cdots\right) \in \mathfrak{a} .
\end{gathered}
$$

Since $\mathfrak{a}$ is index-homogeneous, we have $F^{[-2 \ell]}(\xi) \in \mathfrak{a}(\ell \neq m)$. This shows that we may assume that $F(\xi)$ is an index homogeneous polynomial of index $-2 m$. Putting $s=1$. we have

$$
\begin{aligned}
F\left(\cdots, \sum_{k=0}^{\ell}\left(\begin{array}{l}
\ell \\
k
\end{array}\right) \frac{t^{k}}{w_{j}\left(w_{j}-1\right) \cdots\left(w_{j}-\ell+1\right)}\left(\frac{d}{d z}\right)^{\ell-k} h_{j}(z), \cdots\right) \\
-F\left(\cdots, \frac{1}{w_{j}\left(w_{j}-1\right) \cdots\left(w_{j}-\ell+1\right)}\left(\frac{d}{d z}\right)^{\ell} h_{j}(z) . \cdots\right)=0 .
\end{aligned}
$$

The coefficient of $t$ in this equation must be zero, and it is nothing else than

$$
(\mathscr{D F})\left(\cdots, \frac{1}{w_{j}\left(w_{j}-1\right) \cdots\left(w_{j}-\ell+1\right)}\left(\frac{d}{d z}\right)^{\ell} h_{j}(z), \cdots\right)=0 .
$$


This shows that $\mathscr{D} F(\xi) \in a$, since $w_{1}, \cdots, w_{N}$ are negative integers, Condition (C) in $\S 2$ is satisfied by $w_{1}, \cdots, w_{N}$. Hence by virtue of Theorem 1.2 there exist binary semi-invariants $\varphi_{0}(\xi), \cdots, \varphi_{m-1}(\xi)$ of index $-2 m$, $-2(m-1), \cdots,-2$ such that

$$
F(\xi)=\sum_{k=0}^{m-1} \Delta^{k} \varphi_{k}(\xi)
$$

and $\Delta^{k} \varphi_{k}(0 \leq k \leq m-1)$ are linearly independent. Hence by virtue of (1.3) we have

$$
\begin{aligned}
\Delta \mathscr{D} F(\xi) & =\Delta \mathscr{D} \sum_{k=0}^{m-1} \Delta^{k} \varphi_{k}(\xi)=\Delta \sum_{k=0}^{m-1}\left[\mathscr{D}, \Delta^{k}\right] \varphi_{k}(\xi) \\
& =\sum_{k=0}^{m-1} k(-2(m-k)-k+1) \Delta^{k} \varphi(\xi) \\
& =\sum_{k=0}^{m-1} k(-m+k+1) \Delta^{k} \varphi(\xi),
\end{aligned}
$$

and thus $(\Delta \mathscr{D})^{\ell} F(\xi)=\sum_{k=1}^{m-1}(k(-m+k+1))^{\ell} \Delta^{k} \varphi_{k}(\xi)$. On the other hand by virtue of Proposition 1.6 we have

$$
(\Delta \mathscr{D})^{\ell} F(\xi)=\sum_{k=1}^{m-1}\left(k(-m+k-1)^{\ell} \Delta^{k} \varphi_{k}(\xi) \in \mathfrak{X} \quad(\ell=1,2, \cdots) .\right.
$$

This means

$$
\Delta^{k} \varphi_{k}(\xi) \in \mathfrak{U} \quad(k=1,2, \cdots, m-1),
$$

and thus

$$
F^{*}(\xi) \equiv \varphi_{0}(\xi) \bmod \mathfrak{A}
$$

Namely

$$
\begin{aligned}
& F\left(\frac{(d / d z)^{\ell} h_{j}(z)}{w_{j}\left(w_{j}-1\right) \cdots\left(w_{j}-\ell+1\right)}\right) \\
& \quad=\varphi_{0}\left(\cdots, \frac{(d / d z)^{\ell} h_{j}(z)}{w_{j}\left(w_{j}-1\right) \cdots\left(w_{j}-\ell+1\right)}, \cdots\right)
\end{aligned}
$$

with the semi-invariant $\varphi_{0}(\xi)$.

\section{Chapter 2. Differential invariants of linear ordinary differential operators}

We shall be concerned with linear ordinary differential operators of order $n$ 


$$
L_{n}(p \mid z, y)=\left(\frac{d}{d y}\right)^{n} y+\sum_{\ell=1}^{n}\left(\begin{array}{l}
n \\
\ell
\end{array}\right) p_{\ell}(z)\left(\frac{d}{d z}\right)^{n-\ell} y
$$

where $z$ is a complex independent variable and the coefficients $p_{1}(z), \cdots$, $p_{n}(z)$ are holomorphic at the origin. Similarly as in the case of binary forms, the binomial coefficients $\left(\begin{array}{l}n \\ \ell\end{array}\right)$ play a quite important role.

Pseudo-group $G$ of variable transformations

$$
\rho_{u, \lambda}:(z, y) \longrightarrow(u(z), \lambda(z) y) \quad\left(\frac{d u(0)}{d z} \neq 0, \lambda(0) \neq 0\right)
$$

acts on $L_{n}(p \mid z, y)$ as follows,

$$
\begin{gathered}
L_{n}\left(\rho_{u, \lambda}(p) \mid z, y\right)=\left(\frac{d}{d z}\right)^{n} y+\sum_{\ell=1}^{n}\left(\begin{array}{c}
n \\
\ell
\end{array}\right) \rho_{u, \lambda}(p)_{\ell}(z)\left(\frac{d}{d z}\right)^{n} y \\
=\left(\frac{d u}{d z}\right)^{n} \lambda(z)^{-1} L_{n}(p \mid u(z), \lambda(z) y) .
\end{gathered}
$$

The pseudo-group $G$ acts also naturally on the differential polynomial algebra

$$
C\left[\left(\left(\frac{d}{d z}\right)^{\ell} p_{j}(z)\right)_{1 \leq j \leq n ; \ell=0,1,2, \cdots}\right]
$$

A linear differential operator $L_{n}(Q \mid z, y)$ is called a Laguerre-Forsyth's canonical form if $Q_{1}(z)=Q_{2}(z)=0$.

Forsyth has proved that for each $L_{n}(p \mid z, y)$ there exists a variable transformation $\rho_{u, \lambda}$ such that $L_{n}\left(\rho_{u, \lambda}(p) \mid z, y\right)$ is a Laguerre-Forsyth's canonical form. He also has proved that a variable transformation $\rho_{u, \lambda}$ maps a Laguerre-Forsyth's canonical form to a Laguerre-Forsyth's canonical form, if and only if

$$
u(z)=\frac{\alpha z+\beta}{\gamma z+\delta}, \lambda(z)=\frac{c}{(\lambda z+\delta)^{n-1}},
$$

where

$$
\left(\begin{array}{ll}
\delta & \beta \\
\gamma & \alpha
\end{array}\right) \in S L(2, C), \quad c \neq 0 \in C
$$

A differential polynomial $\varphi\left(\cdots,(d / d z)^{\ell} p_{j}(z) \cdots\right)$ is called a differential invariant of weight $p$, if $\varphi\left(\cdots,(d / d z)^{\ell} p_{j}(z), \cdots\right)(d z)^{p}$ is invariant by the action of $G$. 
Forsyth has found a system of basic differential invariants $\left(\theta_{3}(z), \cdots\right.$, $\left.\theta_{n}(z)\right)$ of $L(p \mid z, y)$ such that i) $\theta_{3}(z), \cdots, \theta_{n}(z)$ are weight $3, \cdots, n$, respectively, ii) if $L_{n}(Q \mid z, y)$ is a Laguerre-Forsyth's canonical form of $L_{n}(p \mid z, y)$, then

$$
\begin{aligned}
\theta_{p}(z)=\frac{1}{2} \sum_{s=0}^{p-3} \frac{(-1)^{s}(p-2) ! p !(2 p-s-2) !}{(p-s-1) !(p-s) !(2 p-s) ! s !}\left(\frac{d}{d z}\right)^{s} Q_{p-s}(z), & \\
& (3 \leq p \leq n)
\end{aligned}
$$

iii) all the differential invariants of $L_{n}(p \mid z, y)$ can be obtained from $\theta_{3}(z), \cdots, \theta_{n}(z)$, by successive making of jacobians, iv) $\theta_{3}(z), \theta_{4}(z), \cdots$ do not depend on $n$, i.e. for $n^{\prime} \geq n\left(\theta_{3}(z), \cdots, \theta_{n}(z)\right)$ is a part of the system of basic differential invariants of

$$
L_{n^{\prime}}(p \mid z, y)=\left(\frac{d}{d z}\right)^{n^{\prime}} y+\sum_{\ell=0}^{n^{\prime}}\left(\begin{array}{c}
n^{\prime} \\
\ell
\end{array}\right) p_{\ell}(z)\left(\frac{d}{d z}\right)^{n,-\ell} y .
$$

In the present chapter first we shall prove that, if we put

$$
\begin{aligned}
\theta_{p}(z) & =\sum_{\ell=0}^{\infty}\left(\begin{array}{c}
w_{p} \\
\ell
\end{array}\right) \alpha_{p}^{(\ell)} z^{\ell} \quad(3 \leq p \leq n), \\
w_{p} & =-2 p
\end{aligned}
$$

then the ring of all the differential invariants of $L_{n}(p \mid z, y)$ is nothing else than the ring of covariants of formal power series $\theta_{3}(z), \cdots, \theta_{n}(z)$ in the sense of chapter 1 , namely $\varphi$ is a differential invariant of $L_{n}(p \mid z, y)$ if and only if there exists a binary semi-invariant $F\left(\cdots, \xi_{j}^{(\ell)}, \cdots\right)$ of index $w_{p}=$ $-2 p$ such that

$$
\varphi=F\left(\cdots, \frac{(d / d z)^{\ell} \theta_{j}(z)}{w_{j}\left(w_{j}-1\right) \cdots\left(w_{j}-\ell+1\right)}, \cdots\right) .
$$

We choose a fundamental solution $\left(\phi_{1}(z), \cdots, \phi_{n}(z)\right)$ of $L_{n}(p \mid z, y)=0$, and consider

$$
z \longrightarrow\left(\phi_{1}(z), \cdots, \phi_{n}(z)\right)
$$

a curve in projective space $\boldsymbol{P}^{n-1}$ of dimension $n-1$. Then $\theta_{3}(z)(d z)^{3}, \cdots$, $\theta_{n}(z)(d z)^{n}$ projective invariants of the portlate of the projective curve.

We shall show an application to automorphic forms as follows; let $\phi_{1}(z), \cdots, \phi_{n}(z)$ be a set of linearly independent integral automorphic forms of index $-2 k$ with respect to a group $\Gamma$, and put 


$$
\begin{aligned}
& w_{\phi}(z)=\left|\begin{array}{c}
\left(\frac{d}{d z}\right)^{n-1} \phi_{1}, \cdots,\left(\frac{d}{d z}\right)^{n-1} \phi_{n} \\
\vdots \\
\phi_{1}, \cdots \cdots \cdots, \phi_{n}
\end{array}\right|, \\
& L_{n}(p \mid z, y)=w_{\phi}(z)^{-1}\left|\begin{array}{ccc}
\left(\frac{d}{d z}\right)^{n} y,\left(\frac{d}{d z}\right)^{n} \phi_{1}, \cdots,\left(\frac{d}{d z}\right)^{n} \phi_{n} \\
\vdots & \vdots & \vdots \\
y, & \phi_{1}, \cdots \cdots, \phi_{n}
\end{array}\right| .
\end{aligned}
$$

Let $\left(\theta_{3}(z), \cdots, \theta_{n}(z)\right)$ be the system of fundamental differential ivariants of $L_{n}(p \mid z, y)$. Then $\theta_{p}(z)$ is a $\Gamma$-automorphic form of index $-2 p$ whose denominator a factor of a power of Wronskian $W_{\phi}(z)$. It is remarkable that the system of automorphic forms $\left(\theta_{3}(z), \cdots, \theta_{n}(z)\right)$ is uniquely determined by the vector space $\sum_{\ell=1}^{n} C \phi_{\ell}(z)$. If $\left\{\phi_{1}(z), \cdots, \phi_{n}(z)\right\}$ is a base of differential forms of $1^{\text {st }}$ kind of an algebraic curve of genus $n$, then $\theta_{3}(z)(d z)^{3}, \cdots, \theta_{n}(z)(d z)^{n}$ are invariants of the function field of the algebraic curve.

\section{§1. Laguerre-Forsyth's canonical forms}

1.1. We shall first sketch the outline of Laguerre-Forsyth's theory of canonical forms.

We denote by $\mathscr{L}(n)$ the complex vector space of all the homogeneous linear differential operators of degree $n$

$$
L_{n}(p \mid z, y)=\left(\frac{d}{d z}\right)^{n} y+\sum_{\ell=1}^{n}\left(\begin{array}{l}
n \\
\ell
\end{array}\right) p_{\ell}(z)\left(\frac{d}{d z}\right)^{n-\ell} y
$$

whose coefficients $p_{1}(z), \cdots, p_{n}(z)$ are holomorphic at the origin. It must be noticed that the highest coefficients of elements of $\mathscr{L}(n)$ are always 1 .

We denote by $G$ the pseudo-group of variable transformations

$$
\rho_{u, \lambda}:(z, y) \longrightarrow(u(z), \lambda(z) y)
$$

such that $u(z)$ and $\lambda(z)$ are holomorphic at the origin and $d u(0) / d z \neq 0$, $\lambda(0) \neq 0$.

We mean by $G_{1}$ the pseudo-subgroup of $G$ consisting of all the dependent variable transformations

$G_{1}=\left\{\left.\rho_{\mathrm{id}, \lambda}\right|_{\lambda(0) \neq 0} ^{\lambda(z)}\right.$ are holomorphic functions at the origin such that $\}$.

Pseudo-subgroup $G_{2}$ of independent variable transformations around the origin means 
$G_{2}=\left\{\left.\rho_{u, 1}\right|_{(d u(0) / d z) \neq 0} ^{u(z)}\right.$ are holomorphic functions at the origin such that $\}$.

$G$ is generated by $G_{1}$ and $G_{2}$.

The action of $G$ on $\mathscr{L}(n)$ is defined by

$$
\begin{gathered}
L_{n}\left(\rho_{u, \lambda}(p) \mid z, y\right)=\left(\frac{d}{d z}\right)^{n} y+\sum_{\ell=1}^{n}\left(\begin{array}{c}
n \\
\ell
\end{array}\right) \rho_{u, \lambda}(p)_{\ell}(z)\left(\frac{d}{d z}\right)^{n-\ell} y \\
=\left(\frac{d u}{d z}\right)^{n} \lambda(z)^{-1}\left\{\left(\frac{d}{d u}\right)^{n} y+\sum_{\ell=1}^{n}\left(\begin{array}{l}
n \\
\ell
\end{array}\right) p_{\ell}(u)\left(\frac{d}{d u}\right)^{n-1}(\lambda(z) y)\right\} \\
=\left(\frac{d u}{d z}\right)^{n} \lambda(z)^{-1} L_{n}(p \mid u(z), \lambda(z) y) .
\end{gathered}
$$

The action of $G_{2}$ is rather complicated, but the action of $G_{1}$ is quite simple.

LEMma 2.1

$$
L_{n}\left(\rho_{\mathrm{id}, \lambda}(p) \mid z, y\right)=\left(\frac{d}{d z}\right)^{n} y+\lambda(z)^{-1} \sum_{\ell=1}^{n}\left(\begin{array}{l}
n \\
\ell
\end{array}\right) L_{\ell}(p \mid z, \lambda(z))\left(\frac{d}{d z}\right)^{n-\ell} y .
$$

Proof. From the definition of the action of $\rho_{\mathrm{id}, \lambda}$ it follows

$$
\begin{gathered}
L_{n}\left(\rho_{\mathrm{Id}, \lambda}(p) \mid z, y\right)=\lambda(z)^{-1}\left\{\left(\frac{d}{d z}\right)^{n}(\lambda(z) y)+\sum_{\ell=1}^{n}\left(\begin{array}{c}
n \\
\ell
\end{array}\right) p_{\ell}(z)\left(\frac{d}{d z}\right)^{n-\ell}(\lambda(z) y)\right\} \\
=\left(\frac{d}{d z}\right)^{n} y+\lambda(z)^{-1} \sum_{h=1}^{n}\left\{\left(\begin{array}{l}
n \\
h
\end{array}\right)\left(\frac{d}{d z}\right)^{h} \lambda(z)\right. \\
\left.+\sum_{k=1}^{h}\left(\begin{array}{c}
n \\
h-k
\end{array}\right)\left(\begin{array}{c}
n-h+k \\
k
\end{array}\right) p_{h-k}(z)\left(\frac{d}{d z}\right)^{k} \lambda(z)\right\}\left(\frac{d}{d z}\right)^{n-h} y .
\end{gathered}
$$

Since

$$
\left(\begin{array}{c}
n \\
h-k
\end{array}\right)\left(\begin{array}{c}
n-h+k \\
k
\end{array}\right)=\left(\begin{array}{l}
n \\
k
\end{array}\right)\left(\begin{array}{l}
h \\
k
\end{array}\right)
$$

the coefficient of $(d / d z)^{n-h} y$ in $L_{n}\left(\rho_{\mathrm{id}, \lambda}(p) \mid z, y\right)$ is given by

$$
\begin{aligned}
\lambda(z)^{-1} & \left(\begin{array}{l}
n \\
h
\end{array}\right)\left\{\left(\frac{d}{d z}\right)^{h} \lambda(z)+\sum_{k=1}^{h}\left(\begin{array}{l}
h \\
k
\end{array}\right) p_{h-k}(z)\left(\frac{d}{d z}\right)^{k} \lambda(z)\right\} \\
& =\lambda(z)^{-1}\left(\begin{array}{l}
n \\
h
\end{array}\right) L_{h}(p \mid z, \lambda(z)) .
\end{aligned}
$$

1.2. When $(d / d z)^{\ell} p_{j}(z)(1 \leq j \leq n ; \ell=0,1,2, \cdots)$ are algebraically independent over $C$, we define weight in the algebra of differential polynomials 


$$
\left.C\left[\left(\frac{d}{d z}\right)^{\ell} p_{j}(z)\right)_{1 \leq j \leq n ; \ell=0,1,2, \ldots}\right]
$$

as follows

$$
\text { weight }\left(\left(\frac{d}{d z}\right)^{\ell} p_{j}(z)\right)=j+\ell
$$

Definition 2.1. A linear differential operator $L_{n}(P \mid z, y)$ is called a semi-canonical form if $P_{1}(z)=0$.

Proposition 2.1. For each $L_{n}(p \mid z, y)$ there exists a unique semi-canonical form $L_{n}(p \mid z, y)$ such that

$$
L_{n}(P \mid z, y)=L_{n}\left(\rho_{\mathrm{id}, \lambda}(p) \mid z, y\right)
$$

with an element $\rho_{\mathrm{id}, \lambda}$ in pseudo-group $G_{1}$. Moreover the coefficient $P_{\ell}(z)$ of $(d / d z)^{n-\ell} y$ in $L_{n}(P \mid z, y)$ is expressed as an isobaric polynomial of weight $\ell$ in $(d / d z)^{k} p_{j}(z)(0 \leq k \leq n ; 1 \leq j \leq n)$.

Proof. It is well-known in calculus that $L_{n}\left(\rho_{\mathrm{id}, \lambda}(p) \mid z, y\right)$ is a semicanonical form if and only if

$$
\frac{d \lambda(z)}{d z}+\lambda(z) p_{1}(z)=0
$$

Since the quotient of two solutions of this equation is a constant and for non-zero constants $c$

$$
L_{n}\left(\rho_{\mathrm{id}, c_{\lambda}}(p) \mid z, y\right)=L_{n}\left(\rho_{\mathrm{id}, \lambda}(p) \mid z, y\right),
$$

hence the semi-canonical form

$$
L_{n}(P \mid z, y)=L_{n}\left(\rho_{\mathrm{id}, \lambda}(p) \mid z, y\right)
$$

of $L_{n}(p \mid z, y)$ is unique within $G_{1}$. Again by virtue of

$$
\frac{d \lambda(z)}{d z}=-p_{1}(z) \lambda(z)
$$

there exists an isobaric polynomial $\varphi_{\ell}$ of weight $\ell$ in $(d / d z)^{k} p_{1}(z)(0 \leq k \leq n)$ such that

$$
\lambda(z)^{-1}\left(\frac{d}{d z}\right)^{\ell} \lambda(z)=\varphi_{\ell}
$$

From (2.2) it follows 


$$
\begin{aligned}
P_{\ell}(z) & =\lambda(z)^{-1} L_{\ell}(p \mid z, \lambda(z)) \\
& =\lambda(z)^{-1}\left(\frac{d}{d z}\right)^{\ell} \lambda(z)+\sum_{k=1}^{\ell}\left(\begin{array}{l}
\ell \\
k
\end{array}\right) p_{k}(z) \lambda(z)^{-1}\left(\frac{d}{d z}\right)^{\ell-k} \lambda(z) \\
& =\varphi_{\ell}+\sum_{k=1}^{\ell}\left(\begin{array}{l}
\ell \\
k
\end{array}\right) p_{k} \varphi_{\ell-k} .
\end{aligned}
$$

This shows that $P_{\ell}$ is an isobaric polynomial of weight $\ell$ in $(d / d z)^{k} p_{j}(z)$ $(1 \leq j \leq n ; k=0,1,2, \cdots)$.

Definition 2.2. A differential polynomial $\varphi$ in

$$
C\left[\left(\left(\frac{d}{d z}\right)^{\ell} p_{j}(z)\right)_{1 \leq j \leq n ; \ell=0,1,2, \ldots}\right]
$$

is call a differential semi-invariant, if

$$
\left(\cdots,\left(\frac{d}{d z}\right)^{\ell} \rho_{\mathrm{id}, \lambda}(p)_{j}, \cdots\right)=\left(\cdots,\left(\frac{d}{d z}\right)^{\ell} p_{j}, \cdots\right)
$$

for every $\rho_{\mathrm{id}, \lambda}$ in $G_{1}$.

Proposition 2.2. Let $L_{n}(P \mid z, y)$ be the semi-canonical form of $L_{n}(p \mid z, y)$. The ring of all the differential semi-invariants coincides with

$$
C\left[\left(\left(\frac{d}{d z}\right)^{\ell} p_{j}(z)\right)_{2 \leq j \leq n ; 0,1,2, \ldots}\right] .
$$

Proof. Since the semi-canonical form $L_{n}(P \mid z, y)$ of $L_{n}(p \mid z, y)$ is unique within $G_{1}$. Therefore the coefficients $P_{j}(z)(2 \leq j \leq n)$ are differential semiinvariants of $L_{n}(p \mid z, y)$. Moreover the derivatives $(d / d z)^{\ell} p_{j}(z)(2 \leq j \leq n$; $\ell=0,1,2, \cdots)$ are also differential semi-invariants. The rings of differential invariants of $L_{n}(p \mid z, y)$ and $L_{n}(P \mid z, y)$ coincide, because $L_{n}(p \mid z, y)$ is transformed to $L_{n}(P \mid z, y)$ within $G_{1}$, and the ring of differential semiinvariants of $L_{n}(P \mid z, y)$ is a subring of

$$
C\left[\left(\left(\frac{d}{d z}\right)^{\ell} P_{j}(z)\right)_{2 \leq j \leq n ; \ell=0,1,2, \ldots}\right] .
$$

This proves Proposition 2.2.

Definition 2.3. A linear differential operator $L_{n}(Q \mid z, y)$ is called a Laguerre-Forsyth's canonical form if $Q_{1}(z)=Q_{2}(z)=0$.

1.3. We shall be next concerned with the action of pseudo-group $G_{2}$ 
of independent variable transformations. We shall state two lemmas without proofs.

LEMMA 2.3. ${ }^{2}$ Let $z$ be a function in $u$ and put

$$
\left(\frac{d}{d u}\right)^{m} y=\sum_{k=1}^{m} \frac{A_{m, k}}{k !}\left(\frac{d}{d z}\right)^{k} y \text {. }
$$

Then we have

$$
\frac{A_{m, m-2}}{(m-1) !}=\left(\begin{array}{c}
m \\
3
\end{array}\right)\left(\frac{d}{d u}\right)^{3} z\left(\frac{d z}{d u}\right)^{m-3}+3\left(\begin{array}{c}
m \\
4
\end{array}\right)\left(\left(\frac{d}{d u}\right)^{2} z\right)^{2}\left(\frac{d z}{d u}\right)^{m-4}
$$

Lemma 2.3. ${ }^{3)}$ Putting

$$
\eta=\left(\frac{d z}{d u}\right)^{-1}\left(\frac{d}{d u}\right)^{2} z
$$

we have

$$
\rho_{u, 1}(p)_{1}(z)=\left(\frac{d z}{d u}\right)^{-1}\left(p_{1}(u)+\frac{n-1}{2} \eta\right)
$$

$$
\begin{aligned}
\rho_{u, 1}(p)_{2}(z)=\left(\frac{d z}{d u}\right)^{-2}\{ & p_{2}(u)+(n-2) p_{1}(u) \eta \\
& \left.\quad+\frac{1}{12}\left(\left(3 n^{2}-11 n+10\right) \eta^{2}+\frac{n-2}{3}\right) \eta^{\prime}\right\}
\end{aligned}
$$

$$
\frac{d}{d u}\left(\rho_{u, 1}(p)_{1}(z)\right)=\left(\frac{d z}{d u}\right)^{-2}\left\{p_{1}^{\prime}(u)-p_{1}(u) \eta-\frac{n-1}{2} \eta^{2}+\frac{n-1}{2} \eta^{\prime}\right\},
$$

where ()$^{\prime}=(d / d u)()$.

LEMMA 2.5.

$$
\rho_{u, \lambda o u}(p)_{1}(z)=z^{\prime-1}\left\{p_{1}(u)+\frac{\lambda^{\prime}(u)}{\lambda(u)}+\frac{n-1}{2} \eta\right\},
$$

2) See p. 20-22, § 3 II [4].

3) See (26), (26a), (27) §4 II [4]. 
where ()$^{\prime}=(d / d u)\left(\right.$ ) and $\eta=z^{\prime \prime} / z^{\prime}$.

Proof. From (2.1) it follows

$$
\begin{aligned}
L_{n}\left(\rho_{u, \lambda o u}(p) z, y\right) & =z^{\prime n} \lambda(u)^{-1} L_{n}(p \mid u, \lambda(u) y) \\
& =z^{\prime n} \lambda(u)^{-1}\left\{\left(\frac{d}{d u}\right)^{n}(\lambda(u) y)+\left(\begin{array}{c}
n \\
1
\end{array}\right) p_{1}(u)\left(\frac{d}{d u}\right)^{n-1}(\lambda(u) y)+\cdots\right\} \\
& \left.=z^{\prime n}\left\{\left(\frac{d}{d u}\right)^{n} y+\left(\frac{\lambda^{\prime}(u)}{\lambda(u)}\right)+p_{1}(u)\right)\left(\frac{d}{d u}\right)^{n-1} y+\cdots\right\} .
\end{aligned}
$$

On the other hand from (2.10) it follows

$$
\begin{aligned}
& z^{\prime n}\left\{\left(\frac{d}{d u}\right)^{n} y+\left(\begin{array}{c}
n \\
1
\end{array}\right) p_{1}(u)\left(\frac{d}{d u}\right)^{n-1} y+\cdots\right\} \\
& \quad=\left(\frac{d}{d z}\right)^{n} y+z^{\prime-1}\left\{\left(p_{1}(u)+\frac{\lambda^{\prime}(u)}{\lambda(u)}+\frac{n-1}{2} \eta\right)\left(\frac{d}{d z}\right)^{n-1} y+\cdots\right\},
\end{aligned}
$$

hence

$$
\rho_{u, \lambda \circ u}(p)_{1}(z)=z^{\prime-1}\left\{p_{1}(u)+\frac{\lambda^{\prime}(u)}{\lambda(u)}+\frac{n-1}{2} \eta\right\} .
$$

Proposition 2.2. A transformation in $G$

$$
\rho_{u, \lambda \circ u}:(z, y) \longrightarrow(u, \lambda(u) y)
$$

maps any semi-canonical form to a canonical form, if and only if

$$
\lambda(u)=\frac{c}{(d z / d u)^{n-1}},
$$

where $c$ is a non-zero constant.

Proof. Let $L_{n}(P \mid z, y)$ be a semi-canonical form, i.e. $P_{1}(z)=0$. From (2.13) it follows

$$
\rho_{u, \lambda \circ u}(P)_{1}(z)=z^{\prime-1}\left\{P_{1}(u)+\frac{\lambda^{\prime}(u)}{\lambda(u)}+\frac{n-1}{2} \frac{z^{\prime \prime}}{z^{\prime}}\right\},
$$

hence we observe that

$$
\rho_{u, \text { д० } u}(P)_{1}=0
$$

if and only if

$$
\frac{\lambda^{\prime}(u)}{\lambda(u)}+\frac{n-1}{2} \frac{z^{\prime \prime}}{z^{\prime}}=0
$$


The solutions of this equation are given by

$$
\lambda(u)=\frac{c}{(d z / d u)^{(n-1) / 2}} \quad(c \in C) .
$$

Proposition 2.3. Let $L_{n}(P \mid z, y)$ be a semi-canonical form, and let $L_{n}(Q \mid z, y)$ be the semi-canonical form of $L_{n}\left(\rho_{u, 1}(P) \mid z, y\right)$. Then

$$
Q_{2}(z)=z^{\prime-2}\left\{P_{2}(u)+\frac{n+1}{12} \eta^{2}-\frac{n+1}{6} \eta^{\prime}\right\},
$$

where ()$^{\prime}=d / d u(), \eta=z^{\prime \prime} \mid z^{\prime}$.

Proof. Since $L_{n}(Q \mid z, y)$ is the semi-canonical form of $L_{n}\left(\rho_{u, 1}(P) \mid z, y\right)$, there exists a function $\lambda(z)$ such that

$$
\begin{aligned}
L_{n}(Q \mid z, y) & =L_{n}\left(\rho_{1, \lambda}\left(\rho_{u, 1}(P)\right) \mid z, y\right) \\
& =\lambda(z)^{-1} \sum_{\ell=1}^{n}\left(\begin{array}{l}
n \\
\ell
\end{array}\right) L_{\ell}\left(\rho_{u, 1}(P) \mid z, \lambda(z)\right)\left(\frac{d}{d z}\right)^{n-\ell} y
\end{aligned}
$$

and

$$
\lambda(z)^{-1} \frac{d \lambda(z)}{d z}+\rho_{u, 1}(P)(z)=0
$$

Since

$$
\begin{aligned}
\lambda(z)^{-1}\left(\frac{d}{d z}\right)^{2} \lambda(z) & =\lambda(z)^{-1} \frac{d}{d z}\left(\frac{d \lambda(z)}{d z}\right)=-\lambda(z)^{-1} \frac{d}{d z}\left(\lambda(z) \rho_{u, 1}(P)_{1}\right) \\
& =-\lambda(z)^{-1} \frac{d \lambda(z)}{d z} \rho_{u, 1}(P)_{1}-\frac{d}{d z} \rho_{u, 1}(P)_{1} \\
& =\rho_{u, 1}(P)_{1}^{2}-\frac{d}{d z} \rho_{u, 1}(P)_{1}
\end{aligned}
$$

we have

$$
\begin{aligned}
Q_{2}(z) & =\lambda(z)^{-1} L_{2}\left(\rho_{u, 1}(P) \mid z, \lambda(z)\right) \\
& =\lambda(z)^{-1}\left(\frac{d}{d z}\right)^{2} \lambda(z)+2 \lambda(z)^{-1} \rho_{u, 1}(P)_{1} \frac{d \lambda(z)}{d z}+\rho_{u, 1}(P)_{2} \\
& =\rho_{u, 1}(P)_{2}-\rho_{u, 1}\left(P_{1}\right)^{2}-\frac{d}{d z} \rho_{u, 1}(P)_{1} .
\end{aligned}
$$

Using (2.10), (2.11), (2.12) and $P_{1}=0$, we have

$$
Q_{2}(z)=z^{\prime-2}\left\{P_{2}(u)+\frac{1}{12}\left(3 n^{2}-11 n+10\right) \eta^{2}+\frac{n-2}{3} \eta^{\prime}\right\}
$$




$$
\begin{aligned}
& -z^{\prime-2} \frac{(n-1)^{2}}{4} \eta^{2}-z^{\prime-2}\left\{-\frac{n-1}{2} \eta^{2}+\frac{n-1}{2} \eta^{\prime}\right\} \\
= & z^{\prime-2}\left\{P_{2}(u)+\frac{n+1}{12} \eta^{2}-\frac{n-1}{6} \eta^{\prime}\right\} .
\end{aligned}
$$

Finally we can now prove fundamental theorems due to Forsyth.

Theorem 2.1 (Forsyth). For each $L_{n}(P \mid z, y)$ there exists a transformation; $\rho_{u, \lambda \circ y} ;(z, y) \rightarrow(u, \lambda(u) y)$ such that $L_{n}\left(\rho_{u, \lambda \circ u}(P) \mid z, y\right)$ is a Laguerre-Forsyth's canonical form, i.e. $\rho_{u, \text {, ou }}(P)_{1}=\rho_{u, \text {, o }}(P)_{2}=0$.

Proof. It is enough to start from a semi-canonical form $L_{n}(P \mid z, y)$, i.e. $P_{1}=0$. Let $\rho_{u, \lambda \circ u}:(z, y) \rightarrow(u, \lambda(u) y)$ be a transformation such that

$$
\begin{aligned}
& \lambda(u)=\frac{c}{z^{\prime(n-1) / 2}} \\
& \left(\frac{z^{\prime \prime}}{z^{\prime}}\right)^{1}-\frac{1}{2}\left(\frac{z^{\prime \prime}}{z^{\prime}}\right)^{2}=\frac{6}{n+1} P_{2}(u),
\end{aligned}
$$

where $c$ is a non-zero constant and ()$^{\prime}=(d / d u)()$. By virtue of Proposition 2.2 the first relation implies that $L_{n}\left(\rho_{u, \text {, ou }}(P) \mid z, y\right)$ is a semi-canonical form. From the second relation and (2.13) we have

$$
\rho_{u, \text { } \text { ou }}(P)_{2}=z^{\prime-2}\left\{P_{2}(u)+\frac{n+1}{12}\left(\frac{z^{\prime \prime}}{z^{\prime}}\right)^{2}-\frac{n+1}{6}\left(\frac{z^{\prime \prime}}{z^{\prime}}\right)^{\prime}\right\}=0 .
$$

Hence $L_{n}\left(\rho_{u, \lambda \text {, }}(P) \mid z, y\right)$ is a Laguerre-Forsyth's canonical form.

It must be notice that, to get a Laguerre-Forsyth's canonical form, we need to solve a Ricattis equation

$$
\left(\frac{z^{\prime \prime}}{z^{\prime}}\right)^{\prime}-\frac{1}{2}\left(\frac{z^{\prime \prime}}{z^{\prime}}\right)^{2}=\frac{6}{n+1} P_{2}(u)
$$

in $\boldsymbol{z}^{\prime \prime} / \boldsymbol{z}^{\prime}$.

THEOREM 2.2 (Forsyth). A transformation $\rho_{u, \lambda ० u}$ maps a Laguerre-Forsyth's canonical form to a Laguerre-Forsyth's canonical form, if and only if

$$
\rho_{u, \text { oou }}:(z, y) \longrightarrow\left(\frac{\alpha z+\beta}{\gamma z+\delta}, \frac{c y}{(\gamma z+\delta)^{n-1}}\right) \quad\left(\left(\begin{array}{ll}
\delta & \beta \\
\gamma & \alpha
\end{array}\right) \in S L(2, C), c \neq 0 \in C\right) .
$$

Proof. Let $\rho_{u, i o u}$ be a transformation which maps a Laguerre-Forsyth's canonical form $L_{n}(Q \mid z, y)$ to a Laguerre-Forsyth's canonical form $L_{n}\left(\rho_{u, 2 \circ u}(Q) \mid z, y\right)$. Then, since 


$$
Q_{1}=Q_{2}=\rho_{u, \lambda o u}(Q)_{1}=\rho_{u, \lambda o u}(Q)_{2}=0,
$$

from Proposition 2.2 and Lemma 2.6 we have

$$
\begin{aligned}
& \lambda(u)=\frac{c}{z^{\prime(n-1) / 2}} \\
& \left(\frac{z^{\prime \prime}}{z^{\prime}}\right)^{\prime}-\frac{1}{2}\left(\frac{z^{\prime \prime}}{z^{\prime}}\right)^{2}=0 .
\end{aligned}
$$

The second equation means that the schwarzian $\{z, u\}$ of $z$ with respect to $u$ vanishes. This shows

$$
u=\frac{\alpha z+\beta}{\gamma z+\delta}, \quad \lambda(u)=c\left(\frac{d u}{d z}\right)^{(n-1) / 2}=\frac{c}{(\gamma z+\delta)^{n-1}} .
$$

\section{§2. Differential invariants}

2.1. A linear differential operator $L_{n}(p \mid z, y)$ is called to be algebraically generic, if $(d / d z)^{\ell} p_{j}(z)(1 \leq j \leq n ; \ell=0,1,2, \cdots)$ are algebraically independent over $C$. Such a case each variable transformation $\rho_{u, \lambda}$ induces a differential algebra homomorphism

$$
\begin{aligned}
\rho_{u, 2}: C\left[\left(\left(\frac{d}{d z}\right)^{\ell} p_{j}(z)\right)_{1 \leq j \leq n ; \ell=0,1,2, \ldots}\right] \\
\longrightarrow C\left[\left(\left(\frac{d}{d v}\right)^{\ell} \rho_{u, 2}(p)_{j}(v)\right)_{1 \leq j \leq n ; \ell=0,1,2, \ldots}\right]
\end{aligned}
$$

such that

$$
\rho_{u, \lambda}\left(\left(\frac{d}{d z}\right)^{\ell} p_{j}(z)=\left(\frac{d}{d v}\right)^{\ell} \rho_{u, \lambda}(p)(v) \quad(1 \leq j \leq n ; \ell=0,1,2, \cdots),\right.
$$

where $u \circ v=z$, i.e. $u(v)=z$.

Let us give the rigorous definition of differential invariants of linear differential operators.

Definition 2.4. Let $L_{n}(p \mid z, y)$ be an algebraically generic linear differential operator, and let $m$ be a non-negative integer. A differential invariant of weight $m$ of $L_{n}(p \mid z, y)$ means a polynomial $\varphi\left(\cdots,(d / d z)^{\ell} p_{j}(z), \cdots\right)$ in $(d / d z)^{\ell} p_{j}(z)(1 \leq j \leq n ; \ell=0,1,2, \cdots)$ such that

$$
\begin{array}{r}
\rho_{u, \lambda}\left(\varphi\left(\cdots,\left(\frac{d}{d z}\right)^{\ell} p_{j}(z), \cdots\right)\right)=\left(\frac{d v}{d z}\right)^{-m} \varphi\left(\cdots,\left(\frac{d}{d z}\right)^{\ell} p_{j}(z), \cdots\right) \\
\left(\rho_{u, \lambda} \in G\right),
\end{array}
$$


where $u \circ v=$ id, i.e. $u(v)=z$. Let $L_{n}(q \mid z, y)$ be any linear differential operator. A differential invariant of weight $m$ of $L_{n}(q \mid z, y)$ is the image of a differential invariant $\varphi\left(\cdots,(d / d z)^{\ell} p_{j}(z), \cdots\right)$ of weight $m$ of $L_{n}(p \mid z, y)$ under the specialization:

$$
C\left[\cdots,\left(\frac{d}{d z}\right)^{\ell} p_{j}(z), \cdots\right] \longrightarrow C\left[\cdots,\left(\frac{d}{d z}\right)^{\ell} q_{j}(z), \cdots\right]
$$

such that

$$
\left(\frac{d}{d z}\right)^{\ell} p_{j}(z) \longrightarrow\left(\frac{d}{d z}\right)^{\ell} q_{j}(z) \quad(1 \leq j \leq n ; \ell=0,1,2, \cdots) .
$$

Obviously this definition of differential invariants of $L_{n}(q \mid z, y)$ does not depends on the choice of an algebraical generic $L_{n}(p \mid z, y)$.

LEMMA 2.6. Let $L_{n}(Q \mid z, y)=L_{n}\left(\rho_{u, \text {, ० } u}(p) \mid z, y\right)$ be a Laguerre-Forsyth's canonical form of $L_{n}(p \mid z, y)$, and let $\varphi\left(\cdots,(d / d z)^{\ell} Q_{j}(z), \cdots\right)$ be a polynomial in $(d / d z)^{\ell} Q_{j}(z)(3 \leq j \leq n ; \ell=0,1,2, \cdots)$ such that

$$
\varphi\left(\cdots,\left(\frac{d}{d z}\right)^{\ell} Q_{j}(z), \cdots\right)(d z)^{m}
$$

is invariant with respect to the transformation group

$$
H=\left\{(z, y) \longrightarrow \frac{\alpha z+\beta}{\gamma z+\delta}, \frac{y}{(\gamma z+\delta)^{n-1}} \mid\left(\begin{array}{ll}
\delta & \beta \\
\gamma & \alpha
\end{array}\right) \in S L(2, C)\right\}
$$

Then there exists a polynomial $\phi\left(\cdots,(d / d u)^{\ell} p_{j}(u), \cdots\right)$ in $(d / d u)^{\ell} p_{j}(u)$ $(1 \leq j \leq n ; \ell=0,1,2, \cdots)$ such that

$$
\phi\left(\cdots,\left(\frac{d}{d u}\right)^{\ell} p_{j}(u), \cdots\right)(d u)^{m}=\varphi\left(\cdots,\left(\frac{d}{d z}\right)^{\ell} Q_{j}(z), \cdots\right)(d z)^{m},
$$

where $m$ is a non-negative integer.

Proof. Since the coefficients of the semi-canonical form of $L_{n}(p \mid z, y)$ are expressed as polynomials in $(d / d z)^{\ell} p_{j}(z)(1 \leq j \leq n ; \ell=0,1,2, \cdots)$, we may assume that $L_{n}(p \mid z, y)$ is itself a semi-canonical form, i.e. $p_{1}=0$. Since $L_{n}(Q \mid z, y)=L_{n}\left(\rho_{\mathrm{id}, \lambda}\left(\rho_{u, 1}(p) \mid z, y\right)\right.$, by virtue of Lemma 2.1 we have

(*) $\quad L_{n}(Q \mid z, y)=\left(\frac{d}{d z}\right)^{n} y+\lambda(z)^{-1} \sum_{\ell=1}^{n}\left(\begin{array}{c}n \\ \ell\end{array}\right) L_{\ell}\left(\rho_{u, 1}(p) \mid z, \lambda(z)\right)\left(\frac{d}{d z}\right)^{n-\ell} y$.

Since $L_{n}(p \mid z, y)$ is a semi-canonical form, by virtue of Proposition 2.2 and 2.3 it follows 
$(* *)$

$(* * *)$

$$
\begin{aligned}
& \lambda(z)=c z^{\prime(n-1) / 2}, \\
& \eta^{\prime}=\frac{1}{2} \eta^{2}+\frac{6}{n+1} q_{2}(u),
\end{aligned}
$$

where ()$^{\prime}=(d / d u)()$ and $\eta=z^{\prime \prime} \mid z^{\prime}$.

From $(* *)$ and $(* * *)$ it follows

$$
\frac{d}{d u} \lambda(z)=\frac{n-1}{2} \frac{z^{\prime \prime}}{z^{\prime}} \lambda(z)=\frac{n-1}{2} \eta \lambda(z)
$$

and

$$
\frac{d}{d z}=z^{\prime-1} \frac{d}{d u}
$$

This shows that i) $\lambda(z)^{-1}(d / d u)^{k} \lambda(z)(k=0,1,2, \cdots)$ can be expressed as polynomials in $\eta, p_{2}(u), p_{2}^{\prime}(u), p_{2}^{\prime \prime}(u), \cdots$ and ii) $(d / d z)^{k}=\sum_{\ell=0}^{k} g_{k, \ell}\left(\eta, z^{\prime-1}\right)(d / d u)^{\ell}$ with polynomials $g_{k, \ell}$ in $\eta$ and $z^{\prime-1}$. Hence by virtue of $(*)$ we can express

$$
\varphi\left(\cdots,\left(\frac{d}{d z}\right)^{\ell} Q_{j}(z), \cdots\right)=\sum_{q, r} \phi_{q, r}\left(\cdots,\left(\frac{d}{d u}\right)^{\ell} p_{j}(u), \cdots\right) z^{\prime-q} \eta^{r}
$$

with a finite number of polynomials $\phi_{q, r}$. On the other hand for $\left(\begin{array}{ll}\delta & \beta \\ \gamma & \alpha\end{array}\right) \epsilon$ $S L(2, C)$

$$
\begin{aligned}
& \left(\frac{\alpha z+\beta}{\gamma z+\delta}\right)^{\prime}=\frac{z^{\prime}}{(\gamma z+\delta)^{2}}, \\
& \frac{((\alpha z+\beta) /(\gamma z+\delta))^{\prime \prime}}{((\alpha z+\beta) /(\gamma z+\delta))^{\prime}}=\frac{z^{\prime \prime}}{z^{\prime}}-\frac{2 \gamma z^{\prime 2}}{\gamma z+\delta}=\eta-\frac{2 \gamma z^{\prime 2}}{\gamma z+\delta} .
\end{aligned}
$$

Since $\varphi\left(\cdots,(d / d z)^{\ell} Q_{j}(z), \cdots\right)(d z)^{m}$ is invariant with respect to $H$, putting $s=(\gamma z+\delta)^{2}$ and $t=-2 \gamma z^{\prime 2}(\gamma z+\delta)^{-1}$, we observe that for $\left(\begin{array}{ll}\delta & \beta \\ \gamma & \alpha\end{array}\right) \in S L(2, C)$

$$
\begin{aligned}
\varphi\left(\cdots,\left(\frac{d}{d z}\right)^{\ell} Q_{j}(z), \cdots\right)(d z)^{m} & \\
= & \sum_{q, r} \phi_{q, r}\left(\cdots,\left(\frac{d}{d u}\right)^{\ell} p_{j}(u), \cdots\right) z^{\prime-q} \eta^{r}(d z)^{m} \\
= & \sum_{q, r} \phi_{q, r}\left(\cdots,\left(\frac{d}{d u}\right)^{\ell} p_{j}(u), \cdots\right)\left(\frac{\alpha z+\beta}{\gamma z+\delta}\right)^{\prime}-q \\
& \quad \times\left(\frac{((\alpha z+\beta) /(\gamma z+\delta))^{\prime \prime}}{((\alpha z+\beta) /(\gamma z+\delta))^{\prime}}\right)^{r} d\left(\left(\frac{\alpha z+\beta}{\gamma z+\delta}\right)\right)^{m}
\end{aligned}
$$




$$
=\sum_{q, r} \phi_{q, r}\left(\cdots,\left(\frac{d}{d u}\right)^{\ell} p_{j}(u), \cdots\right) s^{q-m} z^{\prime-q}(\eta-t)^{r}(d z)^{m}
$$

Here we may choose $\gamma$ and $\delta$ such that $s$ and $t$ are algebraically independent over

$$
C\left(\eta, z^{\prime},\left(\left(\frac{d}{d u}\right)^{\ell} p_{j}(u)\right)_{1 \leq j \leq n ; \ell=0,1,2, \ldots}\right)
$$

hence we have $q=m$ and $r=0$. This means

$$
\varphi\left(\cdots,\left(\frac{d}{d z}\right)^{\ell} Q_{j}(z), \cdots\right)(d z)^{m}=\phi_{m, 0}\left(\cdots,\left(\frac{d}{d u}\right)^{\ell} p_{j}(u), \cdots\right)(d u)^{m} .
$$

THEOREM 2.3. Let $L_{n}(Q \mid z, y)=L_{n}\left(\rho_{u, \text {, ० } u}(p) \mid z, y\right)$ be a Laguerre-Forsyth's canonical form of an algebraically generic $L_{n}(p \mid z, y)$. Then a polynomial $\varphi\left(\cdots,(d / d z)^{\ell} Q_{j}(z), \cdots\right)$ is a differential invariant of weight $m$ of $L_{n}(Q \mid z, y)$, if and only if $\varphi\left(\cdots,(d / d z)^{\ell} Q_{j}(z), \cdots\right)(d z)^{m}$ is invariant with respect to the transformation group

$$
H=\left\{(z, y) \longrightarrow\left(\frac{\alpha z+\beta}{\gamma z+\delta}, \frac{y}{(\gamma z+\delta)^{n-1}}\right) \mid\left(\begin{array}{ll}
\delta & \beta \\
\gamma & \alpha
\end{array}\right) \in S L(2, C)\right\} .
$$

Proof. Let $\varphi\left(\cdots,(d / d z)^{\ell} Q_{j}(z), \cdots\right)(d z)^{m}$ be invariant with respect to $H$. Then by virtue of Lemma 2.6 we get a polynomial $\phi\left(\cdots,(d / d u)^{\ell} p_{j}(u), \cdots\right)$ such that

$$
\text { (*) } \quad \phi\left(\cdots,\left(\frac{d}{d u}\right)^{\ell} p_{j}(u), \cdots\right)(d u)^{m}=\varphi\left(\cdots,\left(\frac{d}{d z}\right)^{\ell} Q_{j}(z), \cdots\right)(d z)^{m}
$$

where $\phi$ is uniquely determined. Let $\rho_{w, \mu}$ be any element and put $\rho_{u, \text { lou }} \circ \rho_{w, \mu}^{-1}$ $=\rho_{x, \nu \circ x}$. Then, since $L_{n}(Q \mid z, y)$ is also a Laguerre-Forsyth's canonical form of $L_{n}\left(\rho_{w, \lambda}(p) \mid z, y\right)$, specializing $\left(u, p_{1}(u), \cdots, p_{n}(u)\right) \rightarrow\left(x, \rho_{w, u}(p)_{1}(x), \cdots, \rho_{w, \mu}(p)_{n}(x)\right)$, we get $\phi\left(\cdots,(d / d x)^{\ell} \rho_{w, \mu}(p)_{j}(x), \cdots\right)(d x)^{m}=\varphi\left(\cdots,(d / d z)^{\ell} Q_{j}(z), \cdots\right)(d z)^{m}=$ $\phi\left(\cdots,(d / d u)^{\ell} p_{j}(u), \cdots\right)(d u)^{m}$. This means that $\phi\left(\cdots,(d / d u)^{\ell} p_{j}(u), \cdots\right)$ is a differential invariant of weight of $L_{n}(p \mid u, y)$. Moreover, specializing $\left(u, p_{1}(u), \cdots, p_{n}(u)\right) \rightarrow\left(z, 0,0, Q_{3}(z), \cdots, Q_{n}(z)\right)$, we get $\phi\left(\cdots,(d / d z)^{\ell} Q_{j}(z)\right.$, $\cdots)(d z)^{m}=\varphi\left(\cdots,(d / d z)^{\ell} Q_{j}(z), \cdots\right)(d z)^{m}$, and thus $\varphi\left(\cdots,(d / d z)^{\ell} Q_{j}(z), \cdots\right)$ is a differential invariant of weight $m$ of $L_{n}(Q \mid z, y)$. Next, we assume conversely that $\varphi\left(\cdots,(d / d z)^{\ell} Q_{j}(z), \cdots\right)$ is a differential invariant of weight $m$ of $L_{n}(Q \mid z, y)$. Then there exists a differential invariant $\phi\left(\cdots,(d / d z)^{\ell} p_{j}(u), \cdots\right)$ of $L_{n}(p \mid u, y)$ such that $\phi\left(\cdots,(d / d z)^{\ell} Q_{j}(z), \cdots\right)=\varphi\left(\cdots,(d / d z) Q_{j}(z), \cdots\right)$. 
Since $\phi\left(\cdots,(d / d u)^{\ell} p_{j}(u), \cdots\right)$ is a differential invariant of weight $m$ of $L_{n}(p \mid u, y)$, we have

$$
\begin{gathered}
\phi\left(\cdots,\left(\frac{d}{d u}\right)^{\ell} p_{j}(u), \cdots\right)(d u)^{m}=\rho_{u, \lambda o u}\left(\phi\left(\cdots,\left(\frac{d}{d u}\right)^{\ell} p_{j}(u), \cdots\right)(d u)^{m}\right) \\
\quad=\phi\left(\cdots,\left(\frac{d}{d z}\right)^{\ell} Q_{j}(z), \cdots\right)(d z)^{m}=\varphi\left(\cdots,\left(\frac{d}{d z}\right)^{\ell} Q_{j}(z), \cdots\right)(d z)^{m} .
\end{gathered}
$$

This shows that $\varphi\left(\cdots,(d / d z)^{\ell} Q_{j}(z), \cdots\right)(d z)^{m}$ is independent on the choice of a Laguerre-Forsyth's canonical form $L_{n}(Q \mid z, y)$. Hence by virtue of Theorem 2.2 it is invariant with respect to $H$.

2.2. We shall first quote a fundamental result on differential invariants due to Forsyth without proof.

TheOREM 2.4) (Forsyth). We denote

$$
\begin{aligned}
\theta_{j}(z)=\frac{1}{2} \sum_{k=0}^{j-3} \frac{(-1)^{k}(j-2) ! j !(2 j-k-2) !}{(j-k-1) !(j-k) !(2 j-3) ! k !}\left(\frac{d}{d z}\right)^{k} Q_{j-k}(z) & \\
& (3 \leq j \leq n)
\end{aligned}
$$

then $\theta_{3}(z)(d z)^{3}, \cdots, \theta_{n}(z)(d z)^{n}$ are invariant with respect to the transformation. group

$$
\left\{(z, y) \longrightarrow\left(\frac{\alpha z+\beta}{\gamma z+\delta}, \frac{c y}{(\gamma z+\delta)^{n-1}}\right) \mid\left(\begin{array}{ll}
\delta & \beta \\
\gamma & \alpha
\end{array}\right) \in S L(2, C), \quad c \neq 0 \in C\right\} .
$$

CoRollaRY. $\theta_{3}(z), \cdots, \theta_{n}(z)$ are differential invariants of $L_{n}(Q \mid z, y)$ of weight $3, \cdots, n$, respectively.

This is an immediate consequence of Theorem 2.3 and Theorem 2.4.

Definition 2.5. $\left(\theta_{3}(z), \cdots, \theta_{n}(z)\right)$ in Theorem 2.4 is called the system of fundamental differential invariants of $L_{n}(Q \mid z, y)$.

Definition 2.6. Let $L_{n}(Q \mid z, y)=L_{n}\left(\rho_{u, 20 u}(q) \mid z, y\right)$ be a Laguerre-Forsyth's canonical form of $L_{n}(q \mid z, y)$. Then there exists a unique system of differential invariants $\left(\theta_{3}(u), \cdots, \theta_{n}(u)\right)$ of $L_{n}(q \mid z, y)$ such that

$$
\theta_{j}(u)(d u)^{m}=\theta_{j}(z)(d z)^{m} \quad(3 \leq j \leq n) .
$$

We call $\left(\theta_{3}(u), \cdots, \theta_{n}(u)\right)$ the system of fundamental differential invariants: of $L_{n}(q \mid u, y)$.

4) See p. 27-32, § 4 II [4]. 
We shall next show a relation between binary semi-invariants in chapter 1 and differential invariants of linear differential operators.

TheOREM 2.5. Let $\xi_{3}=\left(\xi_{3}^{(0)}, \xi_{3}^{(1)}, \cdots\right), \cdots, \xi_{n}=\left(\xi_{n}^{(0)}, \xi_{n}^{(1)}, \cdots\right)$ be vectors of infinite length with variable entries, and put $w_{j}=-2 j(3 \leqq j \leqq n)$. We regard the polynonial ring $C\left[\xi_{3}, \cdots, \xi_{n}\right]$ as an sl(2,C)-admissible ring with respect to $w=\left(w_{3}, \cdots, w_{n}\right)$. Let $L_{n}(Q \mid z, y)$ be a Laguerre-Forsyth's canonical form such that $(d / d z)^{\ell} Q_{j}(z)(3 \leqq j \leqq n ; \ell=0,1,2, \cdots)$ are algebraically independent over $\boldsymbol{C}$. Then a polynomial $\varphi\left(\cdots,\left((d / d z)^{\ell} \theta_{j}(z)\right) /\left(w_{j}\left(w_{j}-1\right) \cdots\left(w_{j}-\ell+1\right)\right)\right)$ is a differential invariant of weight $m$ of $L_{n}(Q \mid z, y)$, if and only if $\varphi\left(\xi_{3}, \cdots, \xi_{n}\right)$ $=\varphi\left(\cdots, \xi_{j}^{(\ell)}, \cdots\right)$ is a binary semi-invariant of index $-2 m$, where $\left(\theta_{3}(z), \cdots\right.$, $\left.\theta_{n}(z)\right)$ is the system of fundamental differential invariants of $L_{n}(Q \mid z, y)$.

Proof. Let $\rho_{u, 2}$ be a variable transformation in $H$;

$$
(z, y) \longrightarrow\left(\frac{\alpha z+\beta}{\gamma z+\delta}, \frac{y}{(\gamma z+\delta)^{n-1}}\right) \quad\left(\left(\begin{array}{ll}
\delta & \beta \\
\gamma & \alpha
\end{array}\right) \in S L(2, C)\right) .
$$

Applying $\rho_{u, \lambda}$ on $\theta_{j}(z)$. we have

$$
\theta_{j}\left(\frac{\alpha v+\beta}{\gamma v+\delta}\right)=\left(\frac{d((\alpha v+\beta) /(\gamma v+\delta))}{d v}\right)^{-j} \rho_{u, \lambda}\left(\theta_{j}\right)(v)=(\gamma v+\delta)^{-w_{j}} \rho_{w, \lambda}\left(\theta_{j}\right)(z),
$$

where $z=(\alpha v+\beta) /(\gamma v+\delta)$. By virtue of Lemma 1.12 we have

$$
\begin{aligned}
& \frac{(d /(d((\alpha v+\beta) /(\gamma v+\delta))))^{\ell} \theta_{j}((\alpha v+\beta) /(\gamma v+\delta))}{w_{j}\left(w_{j}-1\right) \cdots\left(w_{j}-\ell+1\right)} \\
& =\frac{(d /(d((\alpha v+\beta) /(\gamma v+\delta))))^{\ell}(\gamma v+\delta)^{-w_{j}} \rho_{u, 2}\left(\theta_{j}\right)(v)}{w_{j}\left(w_{j}-1\right) \cdots\left(w_{j}-\ell+1\right)} \\
& =(\gamma v+\delta)^{-w_{j}+2 \ell} \sum_{k=0}^{\ell}\left(\begin{array}{l}
\ell \\
k
\end{array}\right) \frac{(d / d v)^{\ell-k} \rho_{u, 2}\left(\theta_{j}\right)(v)}{w_{j}\left(w_{j}-1\right) \cdots\left(w_{j}-\ell+k+1\right)} t^{k},
\end{aligned}
$$

where $t=-\gamma(\gamma v+\delta)^{-1}$ and $\rho_{u, \lambda}$ means the homomorphism

$$
\left(\frac{d}{d z}\right)^{\ell} Q_{j}(z) \longrightarrow\left(\frac{d}{d v}\right)^{\ell} \rho_{u, \lambda}(Q)_{j}(v) \quad(3 \leqq j \leqq n ; \ell=0,1,2, \cdots) .
$$

We first assume that $F\left(\xi_{3}, \cdots, \xi_{n}\right)=F\left(\cdots, \xi_{j}^{(\ell)}, \cdots\right)$ is a binary semiinvariant of index $-2 m$, then we have

$$
\begin{aligned}
& F\left(\cdots, \frac{(d / d z)^{\ell} \theta_{j}(z)}{w_{j}\left(w_{j}-1\right) \cdots\left(w_{j}-\ell+1\right)}, \cdots\right) \\
& \quad=F\left(\cdots, \frac{(d /(d((\alpha v+\beta) /(\gamma v+\delta))))^{\ell} \theta_{j}((\alpha v+\beta) /(\gamma v+\delta))}{w_{j}\left(w_{j}-1\right) \cdots\left(w_{j}-\ell+1\right)}, \cdots\right)
\end{aligned}
$$




$$
\begin{aligned}
& =F\left(\cdots,(\gamma z+\delta)^{-w_{j}+2 \ell} \sum_{k=0}^{\ell}\left(\begin{array}{l}
\ell \\
k
\end{array}\right) \frac{(d / d v)^{\ell-k} \rho_{u, \lambda}\left(\theta_{j}\right)(u)}{w_{j}\left(w_{j}-1\right) \cdots\left(w_{j}-\ell+k+1\right)} t^{k}, \cdots\right) \\
& =\left.(\gamma v+\delta)^{-2 m} F\left(\left(\begin{array}{cc}
1 & 0 \\
t & 1
\end{array}\right) \xi_{3}, \cdots,\left(\begin{array}{cc}
1 & 0 \\
t & 1
\end{array}\right) \xi_{n}\right)\right|_{\xi_{j}^{(\ell)}}=\frac{(d / d v)^{\ell} \rho_{u, \lambda}\left(\theta_{j}\right)(v)}{w_{j}\left(w_{j}-1\right) \cdots\left(w_{j}-\ell+1\right)} \\
& =\left.(\gamma v+\delta)^{-2 m} F\left(\xi_{3}, \cdots, \xi_{n}\right)\right|_{\xi_{j}^{(\ell)}}=\frac{(d / d v)^{\ell} \rho_{u, \lambda}\left(\theta_{j}\right)(v)}{w_{j}\left(w_{j}-1\right) \cdots\left(w_{j}-\ell+1\right)} \\
& =\left(\frac{d((\alpha v+\beta) /(\gamma v+\delta))}{d v}\right)^{m} F\left(\cdots, \frac{(d / d v)^{\ell} \rho_{u, \lambda}\left(\theta_{j}\right)(v)}{w_{j}\left(w_{j}-1\right) \cdots\left(w_{j}-\ell+1\right)}, \cdots\right) \\
& =\left(\frac{d z}{d v}\right)^{m} \rho_{u, \lambda}\left(F\left(\cdots, \frac{(d / d z)^{\ell} \theta_{j}(z)}{w_{j}\left(w_{j}-1\right) \cdots\left(w_{j}-\ell+1\right)}, \cdots\right)\right.
\end{aligned}
$$

This shows that

$$
\text { (*) } \quad F\left(\cdots, \frac{(d / d z)^{\ell} \theta_{j}(z)}{w_{j}\left(w_{j}-1\right) \cdots\left(w_{j}-\ell+1\right)}, \cdots\right)(d z)^{m}
$$

is invariant with respect to $H$, hence

$$
F\left(\cdots, \frac{(d / d z)^{\ell} \theta_{j}(z)}{w_{j}\left(w_{j}-1\right) \cdots\left(w_{j}-\ell+1\right)}, \cdots\right)
$$

is a differential invariant of weight $m$ of $L_{n}(Q \mid z, y)$. We assume next that (*) is invariant with respect to $H$, i.e. $(* *)$ is a differential invariant of weight $m$ of $L(Q \mid z, y)$, then, putting $s=\gamma v+\delta$ and $t=-\gamma(\gamma v+\delta)^{-1}$, we have

$$
\begin{aligned}
s^{2 m} F & \left(\cdots, \frac{(d / d v)^{\ell} \rho_{u, \lambda}\left(\theta_{j}\right)(v)}{w_{j}\left(w_{j}-1\right) \cdots\left(w_{j}-\ell+1\right)}, \cdots\right) \\
& =F\left(\cdots, \frac{(d / d z)^{\ell} \theta_{j}(z)}{w_{j}\left(w_{j}-1\right) \cdots\left(w_{j}-\ell+1\right)}, \cdots\right) \\
& =F\left(\cdots, \frac{(d /(d((\alpha v+\beta) /(\gamma v+\delta))))^{\ell} \theta_{j}((\alpha v+\beta) /(\gamma v+\delta))}{w_{j}\left(w_{j}-1\right) \cdots\left(w_{j}-\ell+1\right)}, \cdots\right) \\
& =F\left(\cdots, s^{2(\ell+j)} \sum_{k=0}^{\ell}\left(\begin{array}{l}
\ell \\
k
\end{array}\right) \frac{(d / d v)^{\ell-k} \rho_{u, \lambda}\left(\theta_{j}\right)(v)}{w_{j}\left(w_{j}-1\right) \cdots\left(w_{j}-\ell+k+1\right)} t^{k}, \cdots\right) .
\end{aligned}
$$

Hence we have a polynomial relation in $s$ and $t$

$$
\begin{gathered}
(* * *) \quad F\left(\cdots, s^{2(\ell+j)} \sum_{k=0}^{\ell}\left(\begin{array}{l}
\ell \\
k
\end{array}\right) \frac{(d / d v)^{\ell-k} \rho_{u, \lambda}\left(\theta_{j}\right)(v)}{w_{j}\left(w_{j}-1\right) \cdots\left(w_{j}-\ell+k+1\right)} t^{k}, \cdots\right) \\
-s^{2 m} F\left(\cdots, \frac{(d / d v)^{\ell} \rho_{u, \lambda}\left(\theta_{j}\right)(v)}{w_{j}\left(w_{j}-1\right) \cdots\left(w_{j}-\ell+1\right)} \cdot \cdots\right)=0
\end{gathered}
$$


We may choose $\gamma$ and $\delta$ such that $s$ and $t$ are algebraically independent over $C\left[\cdots,(d / d v)^{\ell} \rho_{u, \lambda}\left(\theta_{j}\right)(v), \cdots\right]$. If we put $s=1$, then the coefficient of $t$ in $(* * *)$ given by

$$
\begin{aligned}
F_{1}(\cdots, & \frac{(d / d v)^{\ell} \rho_{u, \lambda}\left(\theta_{j}\right)(v)}{w_{j}\left(w_{j}-1\right) \cdots\left(w_{j}-\ell+1\right)} \\
& =\left.D F\left(\xi_{3}, \cdots, \xi_{n}\right)\right|_{\xi_{j}^{(\ell)}}=\frac{(d / d v)^{\ell} \rho_{u, \lambda}\left(\theta_{j}\right)(v)}{w_{j}\left(w_{j}-1\right) \cdots\left(w_{j}-\ell+1\right)},
\end{aligned}
$$

where $D=\sum_{j} \sum_{\ell} \ell \xi_{j}^{(\ell-1)} \partial / \partial \xi_{j}^{(\ell)}$. Since $\theta_{p}(z)-Q_{p}(z)$ is a polynomial in $(d / d z)^{\ell} Q_{j}(z)(3 \leq j \leq p-1 ; \ell=0,1,2, \cdots)$, we have $C\left[\cdots,(d / d z)^{\ell} \theta_{j}(z), \cdots\right)$ $=C\left[\cdots,(d / d z)^{\ell} Q_{j}(z), \cdots\right]$, and thus $(d / d v)^{\ell} \rho_{u, \lambda}\left(\theta_{j}\right)(3 \leq j \leq n ; \ell=0,1,2, \cdots)$ are algebraically independent over $C$. This shows that $D F\left(\xi_{3}, \cdots, \xi_{n}\right)=0$, i.e. $F\left(\xi_{3}, \cdots, \xi_{n}\right)$ is a binary semi-invariant. Putting $t=0$ in $(* * *)$, we have

$$
\begin{aligned}
& F\left(\cdots, s^{-w_{j}+2 \ell} \frac{(d / d v)^{\ell} \rho_{u, \lambda}\left(\theta_{j}\right)(v)}{w_{j}\left(w_{j}-1\right) \cdots\left(w_{j}-\ell+1\right)}, \cdots\right) \\
& \quad=s^{-2 m} F\left(\cdots, \frac{(d / d v)^{\ell} \rho_{u, \lambda}\left(\theta_{j}\right)(v)}{w_{j}\left(w_{j}-1\right) \cdots\left(w_{j}-\ell+1\right)}, \cdots\right)
\end{aligned}
$$

and thus $F\left(\cdots, s^{-w_{j}+2 \ell} \xi^{(\ell)}, \cdots\right)=s^{-2 m} F\left(\cdots, \xi_{j}^{(\ell)}, \cdots\right)$. Namely $F\left(\xi_{3}, \cdots, \xi_{n}\right)$ is a binary semi-invariant of index $-2 m$.

TheOREm 2.6. Let $\left(\theta_{3}(z), \cdots, \theta_{n}(z)\right)$ be the system of fundamental differential invariants of a Laguerre-Forsyth's canonical form $L_{n}(Q \mid z, y)$. Then a polynomial $\varphi\left(\cdots,(d / d z)^{\ell} Q_{j}(z), \cdots\right)$ is a differential invariant of weight $m$ of $L_{n}(Q \mid z, y)$, if and only if it is a covariant of index $-2 m$ of $\left(\theta_{3}(z), \cdots\right.$, $\left.\theta_{n}(z)\right)$ for the action of $S L(2, C)$ with respect to $w=(-6,-4, \cdots,-2 n)$.

Proof. This is an immediate consequence of Theorem 2.5 and Robert's theorem.

2.3. We shall next be concerned with differential invariants of the linear differential equation of automorphic forms.

Theorem 2.7. Let $\Gamma$ be a subgroup of $S L(2, C)$ which acts properly discontinuously on a domain in $C$ containing the origin, and let $\left(h_{1}(z), \cdots\right.$, $\left.h_{n}(z)\right)$ be a system of linearly independent automorphic forms of index $w$ such that $h_{j}(z)(1 \leq j \leq n)$ are holomorphic at $z=0$. We denote 


$$
W_{h}(z)=\left|\begin{array}{cc}
\frac{h_{1}^{(n-1)}}{w(w-1) \cdots(w-n+2)}, \cdots, \frac{h_{n}^{(n-1)}}{w(w-1) \cdots(w-n+2)} \\
\frac{h_{1}^{(n-2)}}{w(w-1) \cdots(w-n+3)}, \cdots, \frac{h_{n}^{(n-2)}}{w(w-1) \cdots(w-n+3)} \\
\vdots \\
h_{1}, & \vdots \\
\cdots & , h_{n}
\end{array}\right|
$$

and

$$
\begin{gathered}
L_{n}(p \mid z, y)=w(w-1) \cdots(w-n+1) W_{h}(z)^{-1} \\
\frac{y^{(n)}}{w(w-1) \cdots(w-n+1)}, \frac{h_{1}^{(n)}}{w(w-1) \cdots(w-n+1)}, \cdots, \frac{h_{n}^{(n)}}{w(w-1) \cdots(w-n+1)} \\
\frac{y^{(n-1)}}{w(w-1) \cdots(w-n+2)}, \frac{h_{1}^{(n-1)}}{w(w-1) \cdots(w-n+2)}, \cdots, \frac{h_{1}^{(n-1)}}{w(w-1) \cdots(w-n+2)} \\
\vdots \\
y, \\
h_{1},
\end{gathered}
$$

where ()$^{(\ell)}=(d / d z)^{\ell}()^{\cdot} \quad$ Let $\left(\theta_{3}(z), \cdots, \theta_{n}(z)\right)$ be the system of fundamental differential invariants of $L_{n}(p \mid z, y)$. Then $\theta_{3}(z), \cdots, \theta_{n}(z)$ are $\Gamma$-automorphic forms of index $-6,-8, \cdots,-2 n$, respectively. If $h_{j}(z)(1 \leq j \leq n)$ are integral, then $W_{h}(z)^{m_{j}} \theta_{j}(z)(3 \leq j \leq n)$ are integral with suitable non-negative integers $m_{j}(3 \leq j \leq n)$.

Proof. Obviously $\left(h_{1}(z), \cdots, h_{n}(z)\right)$ is a fundamental solution of $L_{n}(p \mid z, y)$ $=0$. The Wronskian $W_{h}(z)$ is a $\Gamma$-automorphic form. which is integral provided that $h_{j}(z)(1 \leq j \leq n)$ are integral. Moreover $W_{h}(z) p_{j}(z)(1 \leq j \leq n)$ are expressed as polynomials in $h_{j}^{(\ell)}(z)(1 \leq j \leq n ; \ell=0,1,2, \cdots, n)$; hence it is enough to show

$$
\theta_{j}\left(\frac{\alpha v+\beta}{\gamma v+\delta}\right)=(\gamma v+\delta)^{2 j} \theta_{j}(v) \quad\left(\left(\begin{array}{ll}
\delta & \beta \\
\gamma & \alpha
\end{array}\right) \in \Gamma\right) .
$$

Putting $z=(\alpha v+\beta) /(\gamma v+\delta)\left(\left(\begin{array}{ll}\delta & \beta \\ \gamma & \alpha\end{array}\right) \in \Gamma\right)$, by virtue of Lemma 1.12 we have

$$
\begin{aligned}
& \frac{(d /(d((\alpha v+\beta) /(\gamma v+\delta))))^{\ell}(\gamma v+\delta)^{-w} \mathrm{y}}{w(w-1) \cdots(w-\ell+1)} \\
& \quad=(\gamma v+\delta)^{-w+2 \ell} \sum_{k=0}^{\ell}\left(\begin{array}{l}
\ell \\
k
\end{array}\right) \frac{(d / d v)^{\ell} y}{w(w-1) \cdots(w-\ell+k+1)}\left(-\gamma(\gamma v+\delta)^{-1}\right)^{k}
\end{aligned}
$$

and 


$$
\begin{aligned}
& \frac{(d /(d((\alpha v+\beta) /(\gamma v+\delta))))^{\ell} h_{j}((\alpha v+\beta) /(\gamma v+\delta))}{w(w-1) \cdots(w-\ell+1)} \\
& =\frac{(d /(d((\alpha v+\beta) /(\gamma v+\delta))))^{\ell}(\gamma v+\delta)^{-w} h_{j}(v)}{w(w-1) \cdots(w-\ell+1)} \\
& =(\gamma v+\delta)^{-w+2 \ell} \sum_{k=0}^{\ell}\left(\begin{array}{l}
\ell \\
k
\end{array}\right) \frac{(d / d v)^{\ell-k} h_{j}(v)}{w(w-1) \cdots(w-\ell+k+1)}\left(-\gamma(\gamma v+\delta)^{-1}\right)^{k} .
\end{aligned}
$$

Hence, putting these two relations into $L_{n}\left(p \mid(\alpha v+\beta) /(\gamma v+\delta),(\gamma v+\delta)^{-w} y\right)$, we get

$$
\left(\frac{d((\alpha v+\beta) /(\gamma v+\delta))}{d v}\right)^{n} L_{n}\left(p \mid \frac{\alpha v+\beta}{\gamma v+\delta},(\gamma v+\delta)^{-m} y\right)=(\gamma v+\delta)^{-w} L_{n}(p \mid z, y) .
$$

This means that $\rho_{u, \lambda}(p)_{j}(v)=p_{j}(v)(1 \leq j \leq n)$, where $\rho_{u, \alpha}:(z, y) \rightarrow(\alpha z+\beta) /(\gamma z+\delta)$, $\left.(\gamma z+\delta)^{-m} y\right)$. Since $\theta_{j}(v)$ is a differential invariant of weight $j$ of $L_{n}(p \mid n, y)$, we have

$$
\begin{gathered}
\theta_{j}\left(\frac{\alpha v+\beta}{\gamma v+\delta}\right)\left(d\left(\frac{\alpha v+\beta}{\gamma v+\delta}\right)\right)^{j}=\theta_{j}(z)(d z)^{j} \\
=\rho_{u, \lambda}\left(\theta_{j}\right)(v)(d v)^{j}=\theta_{j}(v)(d v)^{j}
\end{gathered}
$$

and thus

$$
\theta_{j}\left(\frac{\alpha v+\beta}{\gamma v+\delta}\right)=\left(\frac{d z}{d v}\right)^{-j} \theta_{j}(v)=(\gamma v+\delta)^{2 j} \theta_{j}(v) \quad\left(\left(\begin{array}{ll}
\delta & \beta \\
\gamma & \alpha
\end{array}\right) \in \Gamma\right) .
$$

Corollary. Differential invariants of weight $m$ of $L_{n}(p \mid z, y)$ are $\Gamma$ automorphic forms.

Proof. This is an immediate consequence of Theorem 2.6 and Theorem 1.5 .

\section{REFERENCES}

[1] H. Morikawa, Invariant theory (in Japanese), Kinokuniya (1977).

[2] R. A. Rankin, The construction of automorphic forms from the derivatives of a given form, J. Indian Math. Soc. 20 (1956), 103-116.

[ 3 ] I. Schur, Vorlesungen über Invariantentheorie, Springer (1968).

[4] E. J. Wilczynski, Projective differential geometry of curves and ruled surfaces, Chelsea (1961).

[ 5 ] H. Morikawa, On differential invariants of holomorphic projective curves, Nagoya Math. J. 77 (1980), 75-87. 